\title{
Influence of various DEM shape representation methods on packing and shearing of granular assemblies
}

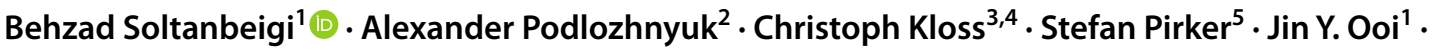 \\ Stefanos-Aldo Papanicolopulos ${ }^{1}$
}

Received: 4 August 2019 / Accepted: 3 December 2020 / Published online: 16 March 2021

(c) The Author(s) 2021

\begin{abstract}
Realistic yet efficient representation of particle shape is a major challenge for the Discrete Element Method. This paper uses angle-of-repose and direct-shear test simulations to describe the influence of several shape representation methods, and their parameters, on the bulk response of granular assemblies. Three rolling resistance models, with varying coefficient of rolling friction, are considered for spherical particles. For non-spherical particles, superquadrics with varying blockiness and multi-spheres with varying bumpiness are used to model cuboids and cylinders of several aspect ratios. We present extensive quantitative results showing how the various ways used to represent shape affect the bulk response, allowing comparisons between different approaches. Simulations of angle-of-repose tests show that all three rolling friction models can model the avalanching characteristics of cube/cuboid and cylindrical particles. Simulations of direct-shear tests suggest that both the shear strength and the dilative response of the considered non-spherical particles (but not their porosity) can only be predicted by the elasto-plastic rolling resistance model. The quantitative nature of the results allows identifying values of the shape-description parameters that can be used to obtain similar results when using alternative shape representation methods.
\end{abstract}

Keywords DEM $\cdot$ Multi-sphere $\cdot$ Superquadrics $\cdot$ Rolling resistance model $\cdot$ Direct shear $\cdot$ Angle of repose

\section{Introduction}

For granular materials, particle shape is an important factor that can highly influence the contact-level behaviour of single particles and, accordingly, affect the bulk-scale response. Non-spherical shapes lead to geometric interlocking between

This article is part of the Topical Collection: Multiscale analysis of particulate micro and macro processes.

Behzad Soltanbeigi

b.soltanbeigi@ed.ac.uk

1 School of Engineering, Institute for Infrastructure and Environment, University of Edinburgh,

Edinburgh EH9 3JL, UK

2 CADS GmbH, Technologiepark 17, 4320 Perg, Austria

3 CFDEMresearch GmbH, Industriezeile 35, 4020 Linz, Austria

4 DCS Computing GmbH, Industriezeile 35, 4020 Linz, Austria

5 Department of Particulate Flow Modelling, Johannes Kepler University, Altenberger Str. 69, 4040 Linz, Austria particles, which hinders the motion of the particles and changes the rheology of the granular assemblies.

Achieving a realistic, yet efficient, representation of particle shape is currently one of the most important challenges for the Discrete Element Method (DEM). In DEM, spheres are usually employed to describe particle shape, due to their simplicity and low computational cost. Restraining rotations through rolling resistance models has been suggested to overcome the inherent inability of the spheres to provide geometric interlocking [14, 37], and thus represent indirectly the effect of particle shape.

A more realistic, but computationally more expensive, alternative is to simulate the particle shape either through rigid clumps of spheres (multi-spheres [12]) or through explicit descriptions of particle shapes (e.g. using polyhedrals, superquadrics or other shapes). While there is extensive literature on particle shape in DEM, we only consider below several studies that have compared the bulk response of various shaped particles, namely spheres with rolling resistance, multispheres, superquadrics, and polyhedrals.

Zhou et al. [36] proposed a methodology to feed DEM simulations with realistic-shaped particles of natural sand. 
They utilized X-ray micro-computed tomography to scan the sand particles. The obtained images are then passed through several image processing techniques, which are followed by the reconstruction of particle surfaces (based on spherical harmonic analysis). The real particle shapes are approximated by clumps of overlapping spheres and fed into DEM simulations. A set of direct shear tests are then performed with the generated complex shape particles and results are compared to spherical assemblies. It is shown that non-spherical particles, due to increased interlocking, yield a higher initial void ratio, peak, and residual strength.

Seelen et al. [27] developed a DEM code to simulate particles of any convex shape. Their study consists of assessing positional and orientational ordering of the generated random granular packings in a cylindrical container. It is observed that the solid fraction is highest in the core of all samples (loose packings of spheres, cuboids, cylinders, and ellipsoids), while it is gradually decreasing towards the boundaries (walls). For cuboids and cylinders, it is shown that the solid fraction smoothly decreases as the aspect ratio $A R$ increases (for $A R>1$ ), while spheres provide the lowest solid fraction. In the case of ellipsoids with aspect ratios higher than one, however, the solid fraction of the samples increases.

Zhao et al. [33] compared the response of spherical particles with rolling resistance to superquadric shape particles (i.e. superballs and ellipsoids) in triaxial compression tests. They showed that increasing shape deviation from the sphere affects the shear strength and dilative response of the granular assembly. Additionally, adjusting rolling resistance can provide a similar influence as the shape parameter on the shear strength, however, it greatly overpredicts the volume change during shearing. They also suggested that the application of rolling resistance, to mimic the behaviour of non-spherical particles, is limited to particle shapes without elongation.

Zhou et al. [34] employed the rolling resistance model proposed for disks by Iwashita and Oda [14], and compared the results with the response of non-spherical particles (triangle and square clumps). They reported that the two approaches present different localisation modes of particle rotation and shear strain at the peak state. The sample with disks shows a clear localization band, while the sample with clump particles exhibits a more uniform localization pattern. They stated that, although the consideration of rolling resistance for disks affects the macroscopic strength and dilatancy of the samples, it is not able to reproduce the rotational behaviour as exhibited by non-spherical particles.

Wensrich and Katterfeld [32] proposed relating the coefficient of rolling friction to a normalized average eccentricity of contact. They evaluated the proposed solution through conducting angle-of-repose tests and compared the results for spheres with rolling resistance, to simple clumped particles. It was seen that similar angle of repose with nonspherical particles can be reached for spheres by considering approximately half of the estimated rolling friction. They suggested that the discrepancy can be justified by considering that the artificial rolling resistance, applied through the proposed methodology, is always acting in the reverse direction of rotation, while real interlocking among particles sometimes can lead to acceleration of the rotation.

Estrada et al. [10] compared the response of discs with rolling resistance, with regular polygonal particles (in 2D). The tests were conducted in simple shear conditions, assessing various aspects of the bulk response. They observed that the rolling friction coefficient can be successfully related to the number of sides in polygonal particles. They also reported that there exists a good agreement between the two types of particle packings in terms of shear strength, solid fraction, force and fabric anisotropy, and probability distribution of contact forces.

In a previous study [28,29], we have compared the influence of multi-sphere bumpiness and superquadric blockiness on the response of assemblies of cubical particles in several micro- and macro-scale tests. We have observed that singleparticle behaviour is a direct function of both surface and edge properties, and that shape complexity only significantly affects the shear strength, porosity and mode of motion once the packing is dense.

A comprehensive comparison between spheres, with restrained rotation, and non-spherical particles is yet missing, with the above-mentioned studies only partially clarifying this issue. We address this by testing various shape complexity factors under various stress states, namely free flowing and dense shearing regimes.

We significantly extend our previous work [29], by considering cylinders in addition to cubes, and varying the particle aspect ratio. Additionally, the present work not only discusses the influence of shape complexity factors, but also specifically focuses on the capability of spheres with rolling friction to simulate characteristics of complex-shaped particles.

The main novelty of the current study is therefore to compare shape representations across multiple aspects, considering different rolling friction models and comparing with different particle shapes and aspect ratios, shape-representation methods, and shape parameters, considering also different testing scenarios and initial densities.

To do so, we simulate angle-of-repose and direct-shear tests to assess the macro-scale influence of several shape complexity factors: rolling friction for spheres (using three models) and shape representation (multi-sphere or superquadric), shape parameter (bumpiness or blockiness), and aspect ratio for cuboids and cylinders. 


\section{Methodology}

This section provides information on the material properties and the testing procedures that have been followed. The considered particles are spheres with $10 \%$ of polydispersity and mean radius of $1 \mathrm{~mm}$ (diameter ranging from $1.9 \mathrm{~mm}$ to $2.1 \mathrm{~mm}$ and following a normal distribution with a mean diameter of $2 \mathrm{~mm}$ and a standard deviation of 0.1 ). Both cubes and cylinders are simulated with various complexities at aspect ratios $(A R)$ between 1 and 3. Additionally, nonspherical particles are monodisperse. The diameter of the spheres is equal to the edge length of non-spheres at $A R=1$.

Cubes/cuboids and cylinders are approximated by two approaches: superquadric (SQ) particles in LIGGGHTS [21, 26] and multi-sphere (MS) particles in EDEM [9]. Regarding the use of two distinct DEM codes, it should be noted that the contact detection algorithm and force calculation methodology are different for multi-sphere and superquadric particles. Furthermore, for modelling spherical particles, both codes are used to consider various rolling resistance models, see Sect. 2.3. The considered rolling resistance models are already implemented in the employed codes and are widely used in the literature.

The parameters for the considered material are chosen so that the computational cost is reasonable for non-spherical particles. Table 1 shows the material properties used in the simulations. The Hertz model with viscous damping [7] is used for normal forces, the Mindlin-Deresiewicz [23] model is used for tangential forces, and no rolling friction model is used for MS and SQ particles.

Anand et al. [3] used DEM to model the discharge of bulk granular materials from a quasi-3D, wedge-shaped hopper. The coefficient of restitution (CoR) was shown to have negligible influence on the discharge rate. Kasina [19] considered four different values of $\operatorname{CoR}\left(10^{-4}, 0.1,0.6\right.$ and 1) and investigated its influence on the bulk internal friction. It is observed that the predicted shear response is insensitive to CoR, since collisions are absent during shearing. Accordingly, as suggested by Kasina [19], a low CoR value of $10^{-4}$ is considered to enhance the settlement of the particle assembly (i.e. to accelerate the process of reaching the steady-state during the filling).

The current study focuses on the impact of various shape complexity factors (i.e. three rolling friction models, bumpiness, blockiness, and aspect ratio). Polydispersity is only considered, to avoid crystallization, for spherical particles. The size distribution is rarely discussed for non-spherical particles in the literature and is not considered in this study. This is to limit the number of parameters for non-spherical particles in the current study. Additionally, the computational cost increases for polydisperse systems, and this is more critical for multispheres, where the time-step is calculated based on diametre of the smallest sub-sphere. For a 2D set of particles, Nguyen et al. [25] presented a detailed numerical investigation of the combined effects of size and shape polydispersity, reporting that triangles and hexagons spontaneously assemble into crystalline structures. However, for square particles with mostly side-side contacts, packings tend to form column-like structures.

\subsection{Superquadrics}

The equation of the shape of a SQ particle in its local coordinate system is [6]:

$f(x, y, z) \equiv\left(\left|\frac{x}{a}\right|^{n_{2}}+\left|\frac{y}{b}\right|^{n_{2}}\right)^{\frac{n_{1}}{n_{2}}}+\left|\frac{z}{c}\right|^{n_{1}}-1=0$,

where $a, b, c$ are the half-lengths of the particle along its principal axes, and $n_{1}$ and $n_{2}$ are parameters that control the shape. For instance, cubical $(a=b=c=d / 2)$ and prolate cuboidal $(a=b=d / 2, c=A R \cdot a)$ particles can be modelled by superquadrics with $n_{1}=n_{2}=N>2$, while prolate cylindrical particles $(a=b=d / 2, c=A R \cdot a)$ can be modelled by taking $n_{1}=N>2, n_{2}=2$, where $N$ controls particle blockiness.

Different levels of blockiness (between $N=4$ and $N=8$, further denoted as $\mathrm{SQ}(N 4), \ldots, \mathrm{SQ}(N 8)$ ) are used in this paper to study the blockiness effect. Fig. 1 (top 2 rows) illustrates particle shapes for $\mathrm{SQ}(N 4), \mathrm{SQ}(N 6)$ and $\mathrm{SQ}(N 8)$ (cubes with $A R=1$ and cylinders with $A R=1.5$ ).

\subsection{Multi-sphere approach}

Multi-spheres, which approximate the shape of particles by overlapping or touching spheres, are used as an approximation of the real shape irregularities [12, 35]. In the multisphere model, a single particle is represented by a set of rigidly connected spheres. The sub-spheres are allowed to vary in size and to overlap forming an approximation of

Table 1 DEM material and simulation properties

\begin{tabular}{ll}
\hline Parameter & Value \\
\hline Density (particle) $\rho\left[\mathrm{kg} / \mathrm{m}^{3}\right]$ & 4100 \\
Coefficient of particle-particle friction $\mu_{s}$ & 0.56 \\
Coefficient of particle-wall friction $\mu_{s \mid p w}$ & 0.45 \\
Coefficient of restitution (particle-particle), $\varepsilon_{p}$ & 0.15 \\
Coefficient of restitution (particle-wall), $\varepsilon_{p w}$ & 0.5 \\
Poisson ratio (particles), $v_{p}$ & 0.25 \\
Poisson ratio (wall), $v_{w}$ & 0.25 \\
Shear modulus (particles), $G_{p}[\mathrm{~Pa}]$ & $10^{7}$ \\
Shear modulus (wall), $G_{w}[\mathrm{~Pa}]$ & $10^{10}$ \\
DEM time-step size, $\Delta t[\mathrm{~s}]$ & $2 \times 10^{-6}$ \\
\hline
\end{tabular}


any desired shape. The contact force between neighbouring particles is calculated from their element spheres, using sphere-sphere contact detection. Details of the algorithm and mechanical calculations can be found in [12].

Cubes, as multi-spheres, were modelled using the EDEM software with equal-radius ( $d / 4)$ overlapping sub-spheres. The number of sub-spheres in each edge of the cubes varies between 2 and 4, resulting in 8, 27 and 64 total sub-spheres per particle, denoted as MS(8), MS(27) and MS(64) correspondingly (see Fig. 1). We have also considered an extension in the number of sub-spheres around a single axis to investigate the effect of the aspect ratio $(A R)$, another shape complexity parameter. Due to an excessive increase in the number of sub-spheres, the extension of MS(64) is not considered (remains with $A R=1$ ). For the angle-of-repose test, $A R$ is increased up to 3 (for both MS(8) and MS(27) particles), while for the direct shear test, the particle extension is considered up to $A R=2$ (due to the high computational time required).

Furthermore, the representation of the cylindrical particles is addressed through considering overlapping spheres, which are aligned along a single axis. The particles consist of 2, 3, 4 and 30 sub-spheres, which leads to variation of the bumpiness. For MS(2), MS(3) and MS(4), the bumpiness is changed along the circumference of the particles, while for MS(30) a top and bottom bumpiness are also added, see Fig. 1. Note that all the non-spherical particles have equal edge length $(2 \mathrm{~mm})$, which is equal to diametre of the simulated spherical particles.

\subsection{Rolling friction models}

Spherical particles have no geometrical interlocking, thus failing for example to capture the formation of stable angles of repose [1]. Consequently, additional rolling friction models $(R F)$ are often used to represent the effects of particle shape. Therefore, when a bulk solid is represented by perfect spheres, the coefficient of rolling friction $\mu_{r}$ is considered as a DEM shape parameter.

Ai et al. [1] reviewed the commonly used rolling friction models proposed in the literature and assessed them in a series of 2D small-scale tests.

The current study uses three rolling resistance models. The first model, called here $\mathrm{RF}_{\mathrm{RVD}}$, is the Relative Velocity Dependent (RVD) rolling friction model in EDEM. It considers equivalent radius $R^{*}$ and unit average rotational velocity vector $\omega_{R V D}$ of the particles.

The $\mathrm{RF}_{\mathrm{RVD}}$ model applies to each particle $i$ in contact with a particle $j$ a torque

$\boldsymbol{T}_{i j(R V D)}=-\mu_{r} R^{*}\left|F_{n}\right|\left(\hat{\boldsymbol{n}}_{i j} \times v_{t_{i j}} /\left|v_{t_{i j}}\right|\right)$

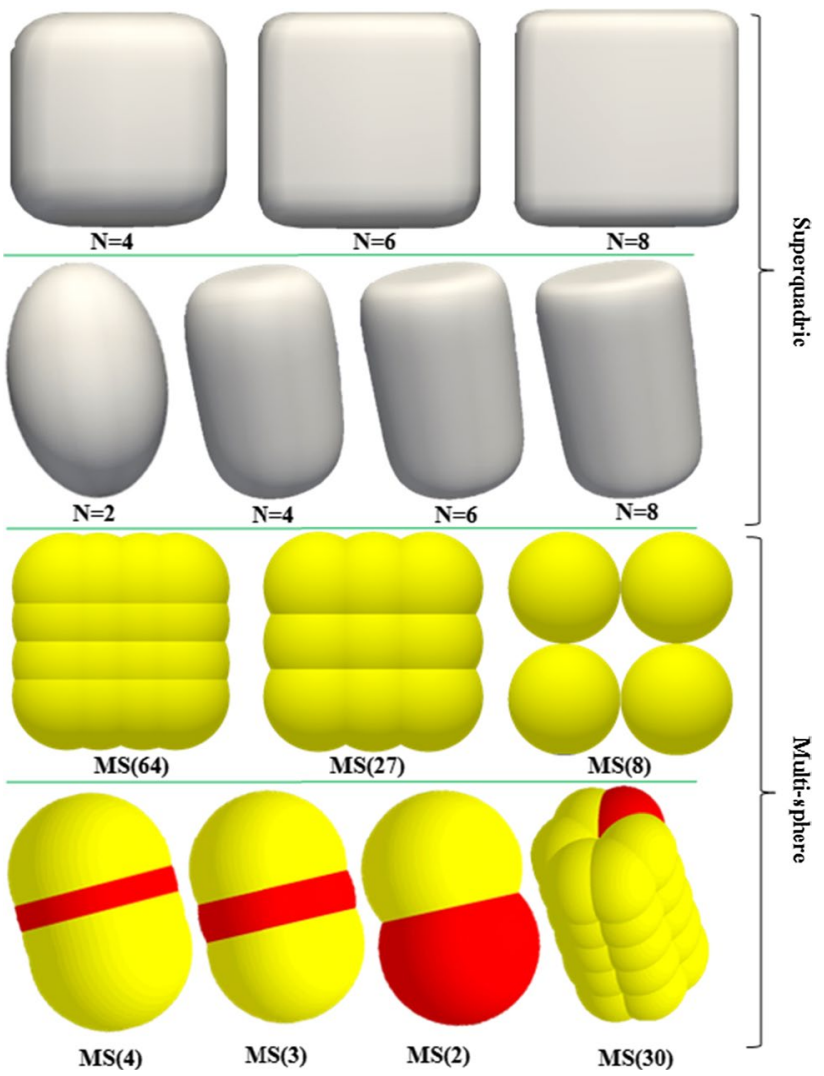

Fig. 1 Non-spherical particles (cubes and cylinders), simulated by means of superquadric (top 2 rows, with increasing blockiness parameter from left to right) and multi-sphere (bottom 2 rows, with increasing bumpiness from left to right) shape representation techniques

where

$R^{*}=\left(R_{i} R_{j}\right) /\left(R_{i}+R_{j}\right)$,

$v_{t_{i j}}=-\frac{1}{2}\left(\omega_{i}+\omega_{j}\right) \times\left(r_{i}-r_{j}\right)$

and $\hat{\boldsymbol{n}}_{i j}$ is a unit vector pointing from particle $i$ to the point of contact.

The second model, called here $\mathrm{RF}_{\mathrm{Cl}}$, is the default rolling resistance model in EDEM, which incorporates the radius $R_{i}$ and unit rotational velocity vector $\hat{\boldsymbol{\omega}}_{i}$ of an individual particle into the torque calculation

$\boldsymbol{T}_{i(C I)}=-\mu_{r} R_{i}\left|F_{n}\right| \hat{\boldsymbol{\omega}}_{i}$

According to the definition by [1], this model is a contactindependent model (type D).

The third model, called here $\mathrm{RF}_{\mathrm{EPSD}}$, is the EPSD2 model in LIGGGHTS [21], which is a modified version of the elastic-plastic spring-dashpot model [14, 16, 17] (type C in [1]). In this modification, the viscous damping term is switched 
off, since it is only effective during dynamic events [32] and to reduce the number of unknown parameters (i.e. rolling viscous damping parameter, which needs to be calibrated [20]). The torque is therefore computed incrementally using only the spring term, as

$\boldsymbol{T}_{r, t+\Delta t}^{k}=\boldsymbol{T}_{r, t}^{k}-k_{r} \Delta \boldsymbol{\theta}_{r}$

where $\Delta \boldsymbol{\theta}_{r}$ is the incremental relative rotation angle between two particles and $k_{r}$ is the rolling stiffness. The magnitude of the spring term is bounded by:

$\left\|\boldsymbol{T}_{r, t+\Delta t}^{k}\right\| \leq \mu_{r} R^{*} F_{n}$.

For determining the $k_{r}$ value, Ai et al. [1] suggested the approach proposed by Bardet and Huang [5]:

$k_{r}=2 J_{n} R^{*} F_{n}$,

where $J_{n}$ is a dimensionless parameter varying between 0.25 and 0.5 [32]; they showed however that, when considering only the spring term, the model is susceptible to oscillations. To overcome this deficiency, Wensrich and Katterfeld [32], recommended the use of the following equation, initially proposed by Iwashita and Oda [14])

$k_{r}=k_{t} R^{* 2}$,

where, $k_{t}$ is the tangential stiffness. Wensrich and Katterfeld [32] pointed out that the rolling stiffness $k_{r}$ calculated from Eq. (9) results in a larger value than from Eq. (8). It is shown that higher $k_{r}$ value leads to mobilization of shear dissipation in contacts, which further hinders the rotation of particles (nullifying the need for a damping term).

As suggested by Jiang et al. [16] the relative rotation of two 3D particles in contact, can be decomposed into two components: a) about the contact normal direction, which renders a twisting resistance torque $b$ ) on the tangential contact plane, which leads to rolling resistance moment. It is shown that both components are influencing the relative motion of the particles, which results in increased dilation (in sample volume) at both triaxial and plane-strain compression tests. However, it is shown that once rolling resistance is excluded from contacts, the strength and dilatancy reduce more (compared to the case where twisting resistance is off), which suggests the importance of rolling resistance. Consequently, in this study, to minimize the number of varying parameters and to highlight the influence of the rolling resistance component, the twisting resistance is not activated.

\section{Simulation of angle-of-repose tests}

\subsection{Test set-up and methodology}

Table 1 shows the properties of the simulated particles. Figure 2 shows the simulation set-up and the dimensions used. The orifice of the funnel has a diameter of $24 \mathrm{~mm}$, which is almost 12 to 4 times the equivalent particle diameter. This ensures free flow of all particles.

Miura et al. [24] observed a decrease in angle of repose (AoR) with increasing number of particles. Wensrich and Katterfeld [32] simulated the AoR test using both clumped particles and spherical particles with rolling friction (7000 particles). Similarly, Chen et al. [8] simulated the AoR test for super-ellipsoids, with 8000 particles. We use here 6000 particles, to avoid excessive computational time.

The particles are distributed randomly in the cone and allowed to settle under gravity for $1 \mathrm{~s}$ simulation time. After the packing is formed, the orifice is opened, and the discharge commences. The simulation continues until a heap is formed, and then the angle of repose is estimated using the algorithm described in [29].

For the spherical assemblies with $\mathrm{RF}_{\mathrm{RVD}}$, special care must be taken for the simulation period after formation of the heap, as particles with $\mathrm{RF}_{\mathrm{RVD}}$ are susceptible to oscillation in static packings [1]. In the angle-of-repose test, after particles are discharged and an initial heap is formed, the rotational and translational velocities tend to reduce, however the constantly generated torque prevents static equilibrium. Applying the artificial torque to the single particles, which are at rest, increases the kinetic energy of the system and this can lead to instability. $\mathrm{RF}_{\mathrm{CI}}$ has a similar mechanism as $\mathrm{RF}_{\mathrm{RVD}}$ for applying the torque, thus facing the same problem of ignoring the particle being static or in motion. Based on our observations and those in [1], this deficiency is more problematic for higher values of $\mu_{r}$ and larger timesteps. Consequently, a small time-step is assigned (1\% of

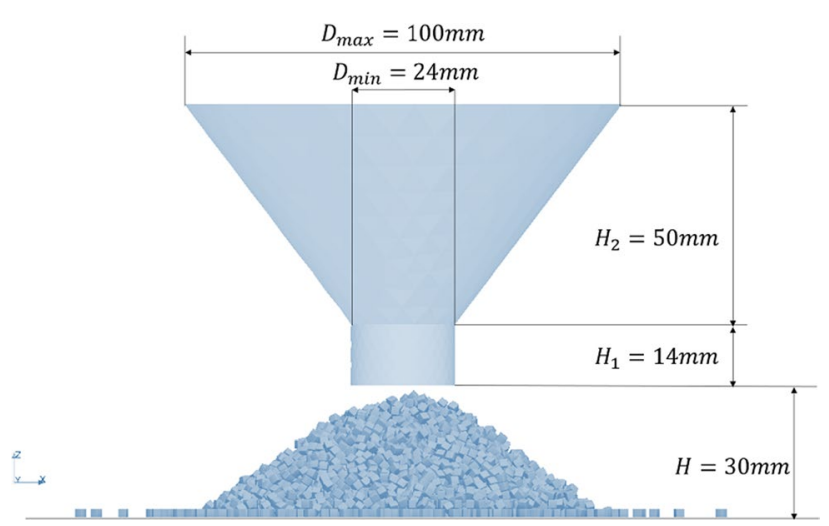

Fig. 2 Angle-of-repose simulation set-up 
the Rayleigh time) for these simulations to minimize the effect of the instabilities. Furthermore, the angle of repose (AoR) is measured at several instances (after the initial heap is formed), to detect any time-dependent changes in the heap geometry. The error bars in Fig. 3 show that for $\mathrm{RF}_{\mathrm{RVD}}$ the difference in AoR is less than $1^{\circ}$ for $\mu_{r} \leq 0.2$, while this difference is less than $2^{\circ}$ for $\mathrm{RF}_{\mathrm{CI}}$ (between $1 \mathrm{~s}$ and $3 \mathrm{~s}$ of simulation time). For larger values of $\mu_{r}$ the overall difference is less than $3^{\circ}$ for both models. Thus, to have consistency between the measured data, all simulations are stopped at exactly $3 \mathrm{~s}$ from the opening of the orifice.

\subsection{Angle or repose results}

Figure 3 shows that the rolling friction models highly affect the heap formation characteristics of the spherical particles. For $\mu_{r} \leq 0.05$ comparable AoR values are obtained for the three models, however by increasing $\mu_{r}, \mathrm{RF}_{\mathrm{CI}}$ leads to larger heap angles compared to those of $\mathrm{RF}_{\mathrm{RVD}}$ and $\mathrm{RF}_{\mathrm{EPSD}}$ (at saturation, the difference is 25\%). The difference in AoR between $\mathrm{RF}_{\mathrm{RVD}}$ and $\mathrm{RF}_{\mathrm{EPSD}}$ is relatively small (especially for $\mu_{r} \leq 0.2$, which is the most frequently used range). The very high AoR for the packings with $\mathrm{RF}_{\mathrm{CI}}$ can be explained by the fact that this model applies the torque to every single particle always in the opposite direction of the rotation (ignoring the relative rotational velocity of the contacting particles).

Another important objective of this study is to evaluate the capability of the rolling resistance models in describing the bulk response of non-spherical particles. Accordingly, we have considered the simulation of non-spherical particles using multi-sphere and superquadric approaches for cubes/ cuboids and cylindrical particle shapes (varying bumpiness and blockiness).

Figure 4 shows the AoR for cube/cuboid shape particles. For $A R=1$, as already reported in [29], increasing

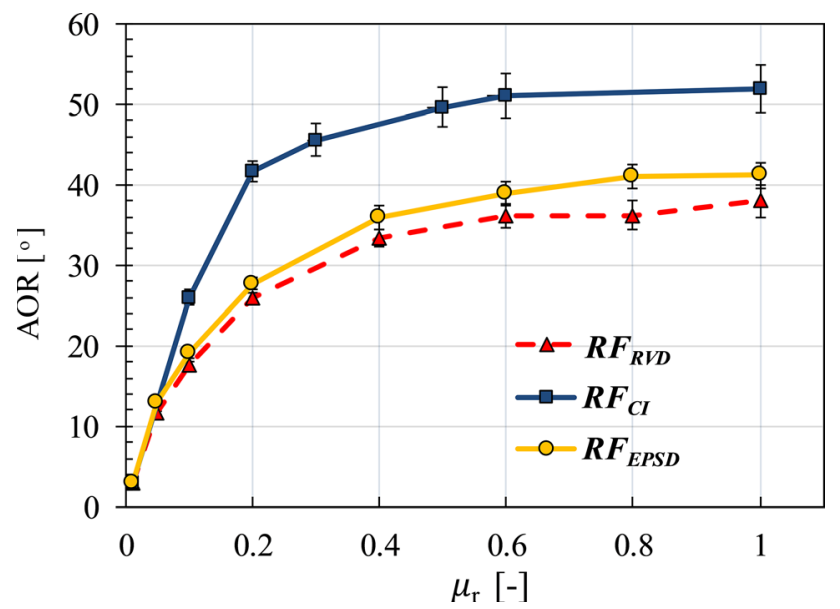

Fig. 3 Angle of repose for spherical particles with different $\mu_{r}$ values blockiness from 4 to 8 contributes to an increase of the AoR of $7^{\circ}$. The increase of bumpiness from MS(64) to MS(27) has an insignificant effect, whereas MS(8), with higher interlocking, provides a larger AoR value. Additionally, varying the aspect ratio from 1 to 3 increases the AoR for all MS and SQ particle types (note that results for MS(64) are only computed for $A R=1$ due to the requirement of high computational time). The results also suggest that, compared to the sharp increase for $\mathrm{SQ}(N 4), \mathrm{SQ}(N 6)$ and $\mathrm{MS}(27)$, the AoR for particles with the highest bumpiness and blockiness are less affected by the increase of $A R$. Another important observation in Fig. 4 is that increasing $A R$ (specially for $A R>2$ ) the difference for the measured AoR between different particles decreases, compared to the difference at $A R=1$.

To further investigate the avalanching characteristics of the non-spherical particles, the AoR test has been also carried out considering cylindrical particles with different aspect ratios (Fig. 5). The first set of tests has been conducted considering SQ particles with different blockiness levels. The results show that increasing the $N$ parameter from 4 to 6 contributes to formation of heaps with higher AoR values (at all aspect ratios). However, cylindrical particles with further blockiness (i.e. $N=8$ and $N=10$ ) do not result in any further significant effect on AoR value (this holds true for all ranges of $A R$ ).

The second set of simulations, for cylindrical particles, has been done using MS particles with varying number of sub-spheres. These tests are only conducted at $A R=1.5$ with particles consisting of 2, 3, 4 and 30 sub-spheres, where the former three types have only bumpiness at the circumference, whereas MS(30) has bumpiness both on its edges (top

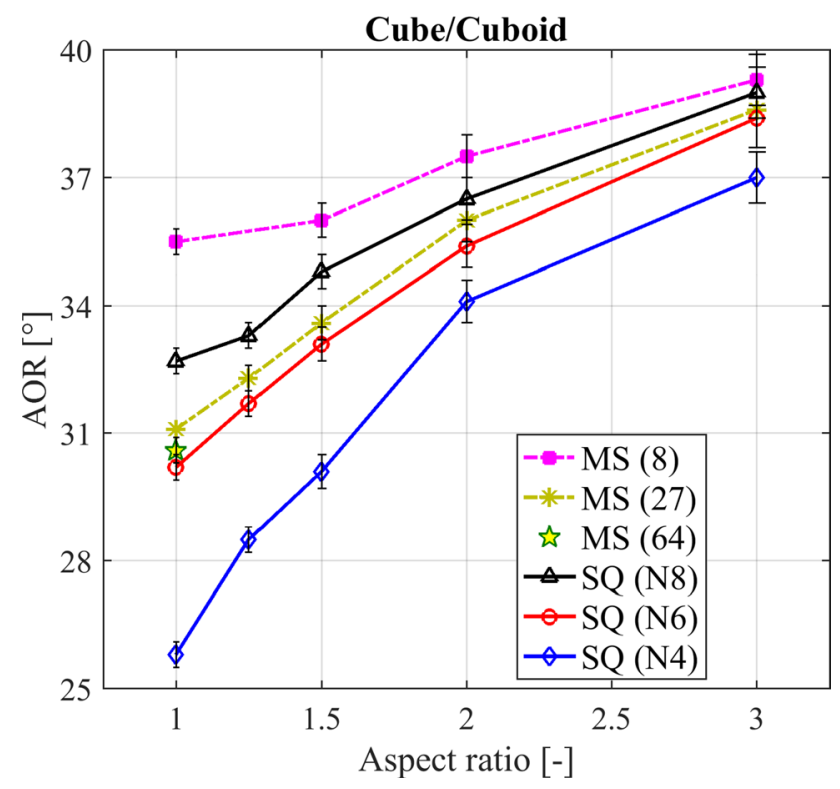

Fig. 4 Angle of repose for cube/cuboid particles 
and bottom) and on its circumference, see Fig. 1. Results for MS particles suggest that increasing the bumpiness at the circumference (from MS(4) to MS(2)) has little influence on the AoR. Increased bumpiness at the edges (i.e. MS(30)) is also required to achieve higher AoR angles. Particles like MS(30) have significantly increased computational cost (both due to increased number of sub-spheres and the need for sub-spheres with smaller radii), whereas changing $N$ has no effect on the computational performance of SQ particles, see [29]. Consequently, the representation of cylinders with MS approach is not extended to other aspect ratios.

\section{Simulation of direct shear test}

This section evaluates the response of granular systems in a shear cell and provides detailed information regarding the influence of different rolling resistance models on initial porosity, shear strength and volume change characteristics of spherical particles. These results are further compared with the response of MS and SQ particles with various shape complexities.

The direct shear test (commonly referred as Jenike shear in bulk solid handling problems) is widely used for measuring flow properties of particulate solids, [13, 30, 36]. In this test, the granular material is placed in a split cylindrical box. Then, the material is compressed by applying a constant vertical load $\sigma_{v}(10 \mathrm{kPa})$ to the lid section (consolidation state). Later, the top half of the cylinder (ring) is sheared at a constant translational velocity $(2 \mathrm{~mm} / \mathrm{s})$, see Fig. 6 . The measured quantity is the force required for this movement

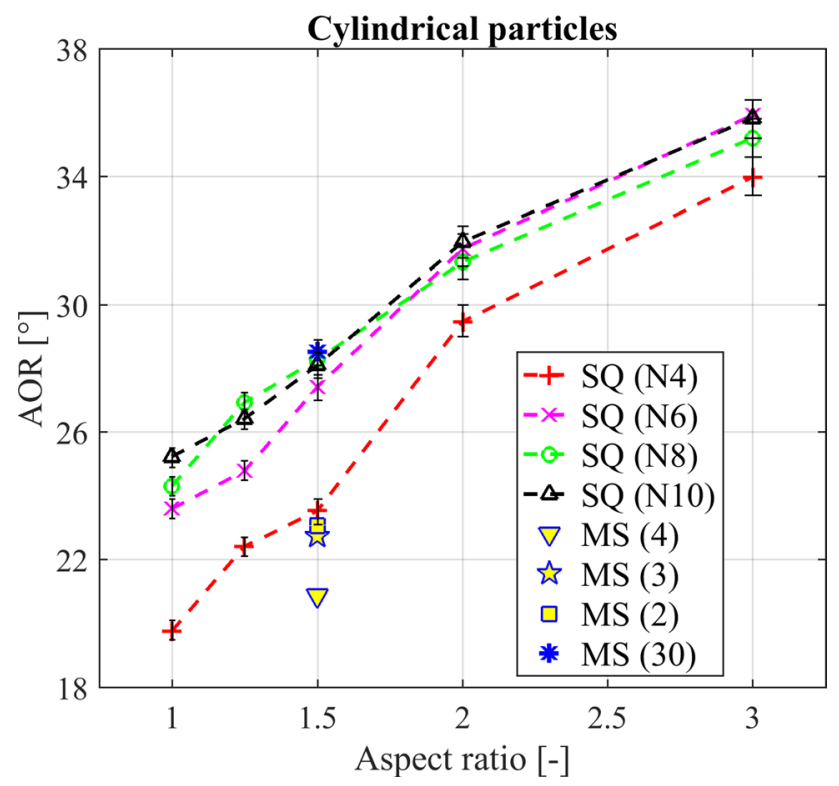

that can be converted to average shear stress $\tau$. The velocity of the lid in LIGGGHTS is controlled by a standard PID controller [2] that compares the current acting force with the predefined target value (in the vertical direction). In EDEM, the position and velocity of the lid are controlled by means of a body-force model written in the API interface of EDEM software.

The DEM time-step was chosen as $\Delta t=2 \times 10^{-6} \mathrm{~s}$ ( $5 \%$ of Rayleigh time) in all simulations.

Two types of packing were generated to assess the dependence of the results on the density state of the initial packing. In the dense packing, the particle-particle sliding friction $\mu_{s}$ and rolling friction $\mu_{r}$ coefficients were set to zero at the filling stage (thus all simulations were carried out based on a single initial packing). Next, before applying $\sigma_{v}$, $\mu_{s}$ is changed back to 0.56 and $\mu_{r}$ is systematically increased for individual packing $\left(\mu_{r}=0.01,0.1,0.2,0.3,0.5,0.8\right.$ and 1). For these packings, the system is allowed to rest for $0.5 \mathrm{~s}$ of simulation time, prior to consolidation. Accordingly, this set of simulations are referred to as equivalent dense initial packing $(E D I P)$, or simply as dense packings.

The sample preparation is carried out by air pluviation. Particles are discharged from a funnel from a certain height to achieve the desired void ratio in the system. To have identical density all over the sample, the factory which generates the particles moves upward with a constant speed. It must be noted that the friction coefficient equals zero for nonspherical particles during the filling.

A second type of packing was established, with $\mu_{s}=0.56$ at all stages of the test. Furthermore, $\mu_{r}$ is applied to the granular assemblies both during filling and shearing (approximating the inclusion of particle shape during the packing). This is done to clarify the importance of the shape parameter (i.e. rolling resistance) in imposing the arrangement of the particles and further evaluate the shearing response of the looser packings (depending on the incorporated value of $\mu_{r}$ ).

For all simulations, material properties were kept identical and packings of equivalent bulk volume were generated for all types of particles (for detailed information regarding the packing density of MS and SQ cubes refer to [29]). Furthermore, Soltanbeigi et al. [29] reported that the shape factor is more influential (on bulk response) in densely packed assemblies, accordingly the cylindrical and cube/ cuboid particles are only simulated in the dense state (i.e. by having zero sliding friction during the filling stage). In the following section (4.1), the effect of including an artificial particle shape parameter on the packing density is evaluated. Successively, the shearing response of the particles is assessed in Sect. 4.3.

Fig. 5 Angle of repose for cylindrical particles 
Fig. 6 Jenike shear tester filled with superquadric particles (dimensions are in millimetres)

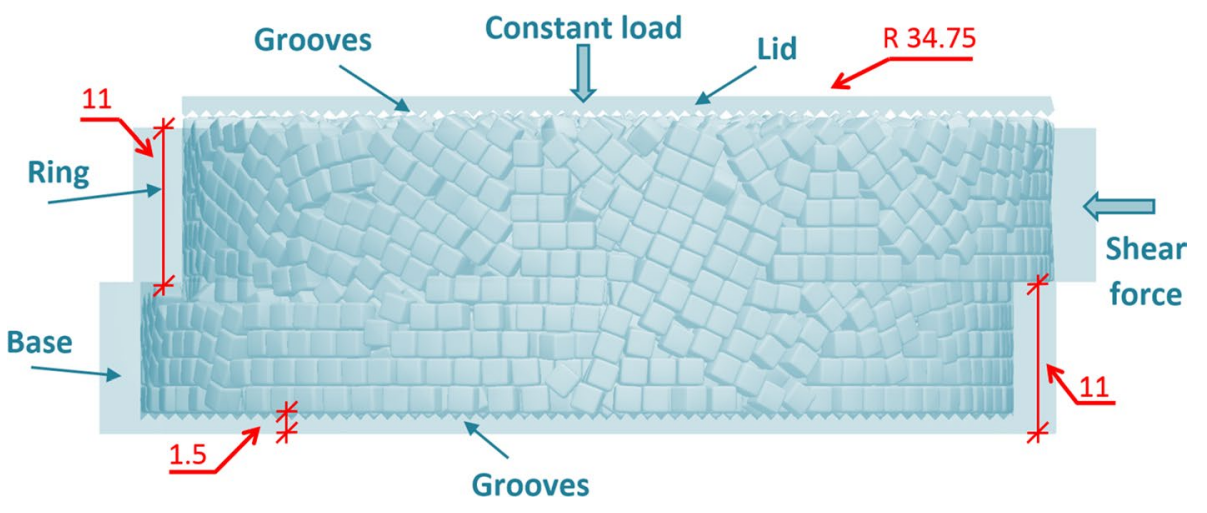

\subsection{Initial porosity}

It is known that applying any type of rolling resistance model affects particle arrangement, and thus the porosity of the granular packing (this is an inherent feature since all models reduce the movement of single particles). Accordingly, the initial porosity $\left(\phi_{\text {init }}\right)$ of the samples was measured before consolidation, by dividing the total volume of the voids over the volume of the shear tester, as an indication of how densely the particles are packed in the system. As mentioned before, the packings are prepared in dense and loose states (by turning on or off $\mu_{s}$ and $\mu_{r}$ ).

There are many studies in the literature for determining the packing characteristics of spherical particles with various properties. For the spherical particles (using DEM), Jerier et al. [15] varied the particle radius (ratio of the largest radius to the smallest one is changed between 2 and 7) and determined a solid fraction within 0.58 to 0.75 (corresponding to $0.25<\phi_{\text {init }}<0.42$ ). Aste [4] reported that pouring mono-sized spheres into a container, typically a solid fraction between 0.61 and $0.62\left(\phi_{\text {init }}=0.38\right.$ and 0.39$)$ is obtained (depending on the geometry of the container, speed and height of filling). It is mentioned that slightly denser packings (i.e. solid fraction of 0.63) can be obtained by gently tapping the container. Additionally, it is reported that to achieve the random close packing limit (i.e. solid fraction of 0.64), the sample must be simultaneously tapped and compressed.

Figure 7 shows porosity for different values of the rolling friction coefficient, for each of the three models. The sliding friction is only changed once, from 0 to 0.56 and then it is kept constant. For dense packings, $\phi_{\text {init }}$ is shown as a single point in Fig. $7\left(\mu_{s}=\mu_{r}=0\right)$, which has the lowest value as $\phi_{\text {init }}=0.377$ (this is in line with results from [4]). Additionally, it is observed that enabling the sliding friction (i.e. $\mu_{s}=0.56$ ) affects the $\phi_{\text {init }}$ (loosens the packing to $\left.\phi_{\text {init }}=0.4\right)$. Moreover, considering higher $\mu_{r}$ values, further increases the porosity in the granular system (compared to the case with no rolling resistance, the maximum difference is about $20 \%$ ). For $\mathrm{RF}_{\mathrm{RVD}}, \phi_{\text {init }}$ increases linearly, while its values are smaller than for the other two models (for the full range of $\mu_{r}$ ). Additionally, for $\mu_{r}<0.2$ the $\mathrm{RF}_{\mathrm{EPSD}}$ model provides the highest $\phi_{\text {init }}$ values (i.e. looser packings). For $\mu_{r} \geq 0.2$, results of $\mathrm{RF}_{\mathrm{CI}}$ and $\mathrm{RF}_{\mathrm{EPSD}}$ are almost comparable (i.e. both models have similar influence on packing, despite their different rotation retardation mechanisms).

Overall, all three models are seen to be effective in governing the particle motion and thus influence the particle arrangement. Additionally, it is clear from the comparison of Figs. 7 and 8 that spheres always reveal larger porosities compared to non-spherical particles. Ma et al. [22] simulated a monosize pack of spheres and varied rolling friction between 0 and 0.2 . It is shown that the initial porosity is increased as rolling friction approximates the particle shape effect and enhances inter-particle interlocking.

Values of $\phi_{\text {init }}$ for particles with various shapes are also computed, and summarized in Fig. 8 (results of cubes with $A R=1$ have already been discussed in [29]). For SQ cuboids, it is seen that increasing aspect ratio leads to slightly increased $\phi_{\text {init }}$ for $\mathrm{SQ}(N 6)$ and $\mathrm{SQ}(N 8)$ with $A R=1.25$, while $\mathrm{SQ}(N 4)$ is independent of the $A R$ value,

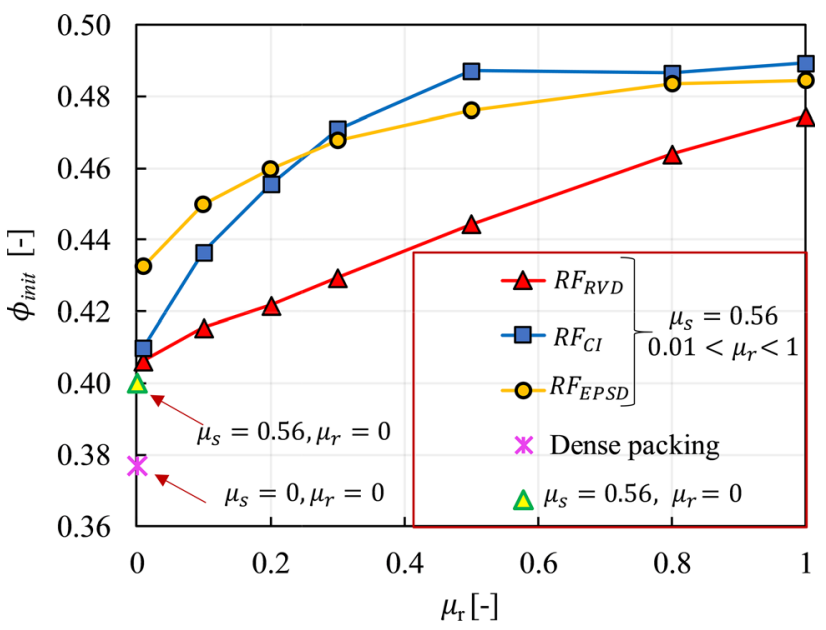

Fig. 7 Initial porosity of the packings with spherical particles in the presence of rolling friction (loose packings) 
see Fig. 8a. For MS particles, the sharpest increase in $\phi_{\text {init }}$ is seen for the MS(8) particle. However, MS(27) shows almost a constant value for $\phi_{\text {init }}$ (a slight increase for $\phi_{\text {init }}$ is seen while moving from $A R=1$ to $A R=1.25$ ). Note that, similar to Sect. 3, MS(64) is not simulated for $A R>1$.

Furthermore, the results for cylindrical particles are plotted in Fig. 8b. A cylindrical particle with $N=2$ would represent a sphere if $A R=1$, while ellipsoids are formed for $A R>1$. It is clearly seen that the initial porosity is affected once the spheres are converted to ellipsoids. In general, it can be suggested that cylinders, at any level of shape complexity, have lower porosity than spheres. The difference in porosity for spheres (with $\mu_{r}=0$ and $\mu_{s}=0.56$ ) in Figs. 7 and $8 \mathrm{~b}$ can be justified by the fact that the spheres generated by the superquadric approach are mono-sized (thus have larger $\phi_{\text {init }}$ ). Moreover, for SQ(N4), SQ(N6) and SQ(N8) the aspect ratio parameter is not affecting the packing porosity (at $A R>1.5$, identical $\phi_{\text {init }}$ values are obtained for all $N$ values). Information regarding particle number and porosity is summarized in Table 2.

Finally, the packing characteristics of spherical particles (in the presence/absence of rolling resistance models), are compared to those of cuboids and cylinders through assessing Figs. 7 and 8. It can be pointed out that except for MS(8), which has an extra porosity between adjacent sub-spheres, all cubes/cuboids and cylinders (in dense state) have lower porosities than spheres.

\subsection{Volume change during shearing}

It is also useful to monitor the vertical displacement of the lid, during the shearing of the granular assemblies, to determine the mode of volume change (i.e. contractive or dilative).

For the dense packings, as observed in Fig. 9, for all three rolling friction models contraction of the assemblies is insignificant and dilation is the dominant mode of volume change. The packings with $\mathrm{RF}_{\mathrm{RVD}}$ experience similar lid displacement and are thus almost independent of the $\mu_{r}$ value (at the shearing stage).

For a certain range of rolling friction coefficients (i.e. $0.01<\mu_{r}<0.3$ ) the packings with $\mathrm{RF}_{\mathrm{CI}}$ follow a similar trend compared to $\mathrm{RF}_{\mathrm{RVD}}$. However, for $\mu_{r}>0.3$, the vertical displacement $D_{L}$ decreases slightly (i.e. the system starts to dilate to a lesser extent). It is already seen in Sect. 3 that $\mathrm{RF}_{\mathrm{CI}}$ can provide comparable AoR to those of non-spherical particles by assigning $\mu_{r}<0.18$. Accordingly, it can be suggested that in the case of $\mathrm{RF}_{\mathrm{Cl}}$, the value of $\mu_{r}$ has to be less than 0.2 , to get reasonable results.

For $\mathrm{RF}_{\mathrm{CI}}$ with $\mu_{r}>0.3$ sliding starts to happen among most of contacts, and accordingly the packing experiences less dilation. A high value of $\mu_{r}$ or $\mu_{s}$ nullifies the respective degree of freedom, and therefore contributes to occurrence

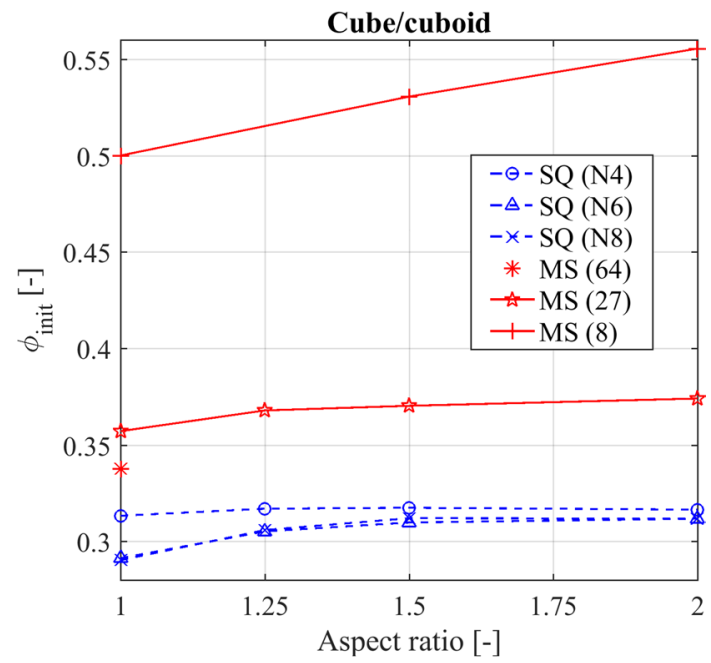

(a)

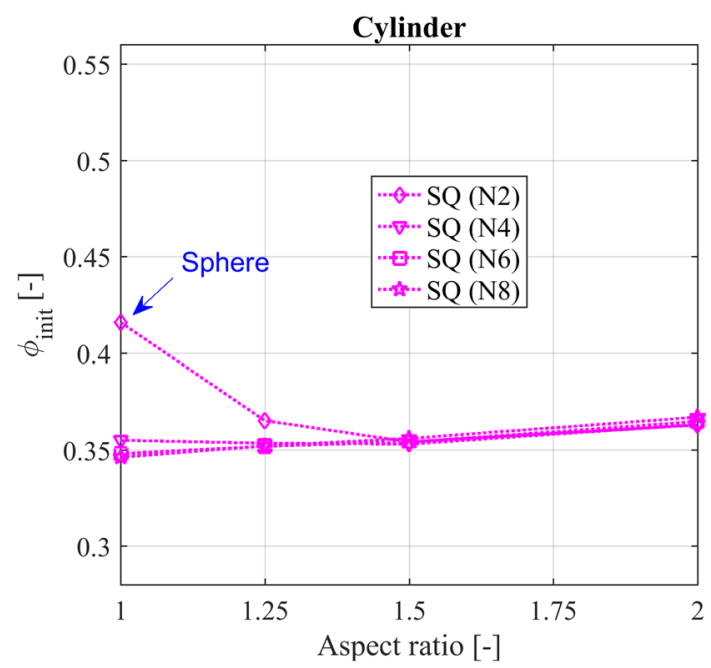

(b)

Fig. 8 Initial porosity of the packings with $\mathbf{a}$ cube/cuboid $\mathbf{b}$ cylindrical particles

of alternative mode of relative motion. A similar observation is reported by Estrada et al. [11], in which they simulated shearing of a polydisperse system under simple shear conditions and systematically varied sliding and rolling friction coefficients. Their results suggested that when sliding friction is high enough, while $\mu_{r}$ is constant, the dominant mode of motion is contact rolling (the reverse case is also valid, where sliding occurs for major portion of the mobilized contacts).

For packings with $\mathrm{RF}_{\mathrm{EPSD}}$, it can be seen that increasing rolling resistance has a more significant impact on the increase of the lid's vertical movement (i.e. $D_{L}$, in this case, can be 2 times larger than that of for $\mathrm{RF}_{\mathrm{RVD}}$ and $\mathrm{RF}_{\mathrm{CI}}$ ).

For relatively loose packings (with enabled $\mu_{s}$ and $\mu_{r}$ for both filling and shearing), $\mathrm{RF}_{\mathrm{RVD}}$ shows a contractive 
Fig. 9 Vertical displacement of the lid during shearing for dense packings a $\mathrm{RF}_{\mathrm{RVD}} \mathbf{b} \mathrm{RF}_{\mathrm{CI}} \mathbf{c} \mathrm{RF}_{\mathrm{EPSD}}$

behaviour (i.e. the samples follow the characteristics of a loose assembly) for almost all applied $\mu_{r}$, see Fig. 10a. It is only the packing with a very small value of rolling friction coefficient (i.e. $\mu_{r}=0.01$ ) that undergoes a volume reduction

Table 2 Number of particles in the direct shear test

\begin{tabular}{|c|c|c|c|}
\hline Particle & $\mathrm{AR}$ & $\begin{array}{l}\text { Number of par- } \\
\text { ticles }\end{array}$ & Initial porosity \\
\hline \multicolumn{4}{|l|}{ Cuboids } \\
\hline \multirow[t]{4}{*}{$\mathrm{SQ}(N 4)$} & 1 & 6163 & 0.314 \\
\hline & 1.25 & 5871 & 0.317 \\
\hline & 1.5 & 4889 & 0.318 \\
\hline & 2 & 3672 & 0.318 \\
\hline \multirow[t]{4}{*}{$\mathrm{SQ}(N 6)$} & 1 & 6848 & 0.291 \\
\hline & 1.25 & 5371 & 0.305 \\
\hline & 1.5 & 4446 & 0.31 \\
\hline & 2 & 3325 & 0.31 \\
\hline \multirow[t]{4}{*}{$\mathrm{SQ}(N 8)$} & 1 & 6576 & 0.291 \\
\hline & 1.25 & 5145 & 0.306 \\
\hline & 1.5 & 4224 & 0.316 \\
\hline & 2 & 3188 & 0.312 \\
\hline \multirow[t]{3}{*}{$\operatorname{MS}(8)$} & 1 & 7463 & 0.5 \\
\hline & 1.5 & 4671 & 0.53 \\
\hline & 2 & 3317 & 0.555 \\
\hline \multirow[t]{3}{*}{$\operatorname{MS}(27)$} & 1 & 6975 & 0.357 \\
\hline & 1.5 & 4401 & 0.37 \\
\hline & 2 & 3718 & 0.374 \\
\hline $\operatorname{MS}(64)$ & 1 & 6920 & 0.34 \\
\hline \multicolumn{4}{|l|}{ Cylinders } \\
\hline \multirow[t]{4}{*}{$\mathrm{SQ}(N 2)$} & 1 & 9705 & 0.416 \\
\hline & 1.25 & 8445 & 0.365 \\
\hline & 1.5 & 7160 & 0.354 \\
\hline & 2 & 5297 & 0.363 \\
\hline \multirow[t]{4}{*}{$\mathrm{SQ}(N 4)$} & 1 & 8181 & 0.355 \\
\hline & 1.25 & 6561 & 0.354 \\
\hline & 1.5 & 5471 & 0.363 \\
\hline & 2 & 4039 & 0.318 \\
\hline \multirow[t]{4}{*}{$\mathrm{SQ}(N 6)$} & 1 & 7732 & 0.348 \\
\hline & 1.25 & 6149 & 0.352 \\
\hline & 1.5 & 5107 & 0.354 \\
\hline & 2 & 3767 & 0.364 \\
\hline \multirow[t]{4}{*}{$\mathrm{SQ}(N 8)$} & 1 & 7548 & 0.346 \\
\hline & 1.25 & 5984 & 0.352 \\
\hline & 1.5 & 4957 & 0.356 \\
\hline & 2 & 3654 & 0.367 \\
\hline $\operatorname{MS}(2)$ & 1.5 & 8948 & 0.338 \\
\hline $\operatorname{MS}(3)$ & 1.5 & 8861 & 0.327 \\
\hline MS(4) & 1.5 & 8801 & 0.328 \\
\hline $\operatorname{MS}(30)$ & 1.5 & 7698 & 0.373 \\
\hline
\end{tabular}

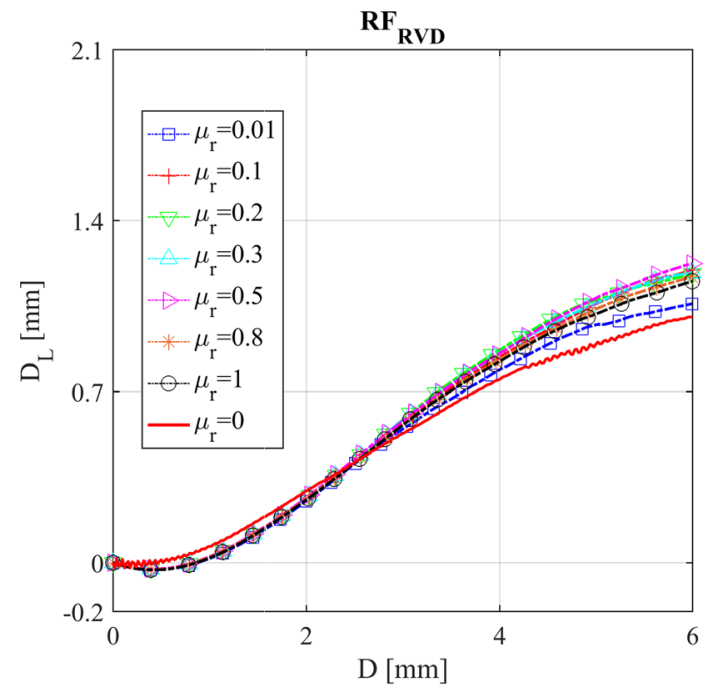

(a)

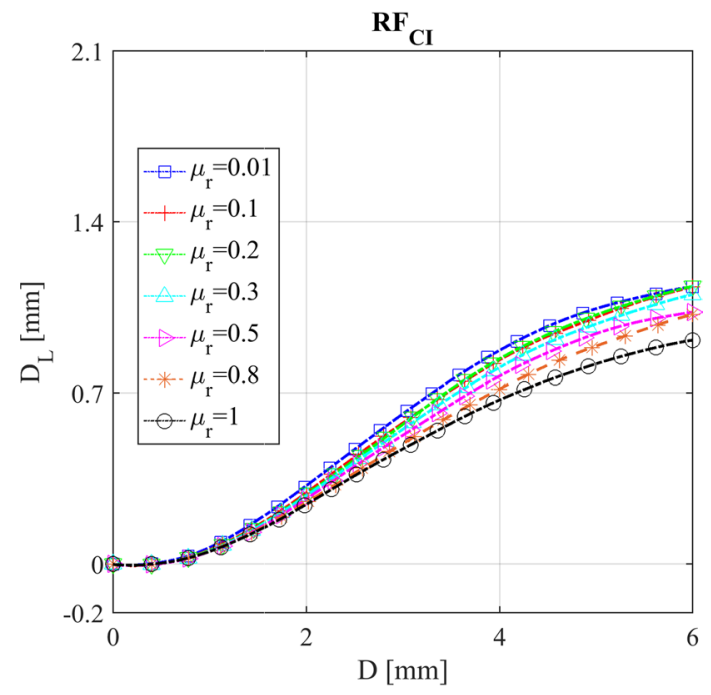

(b)

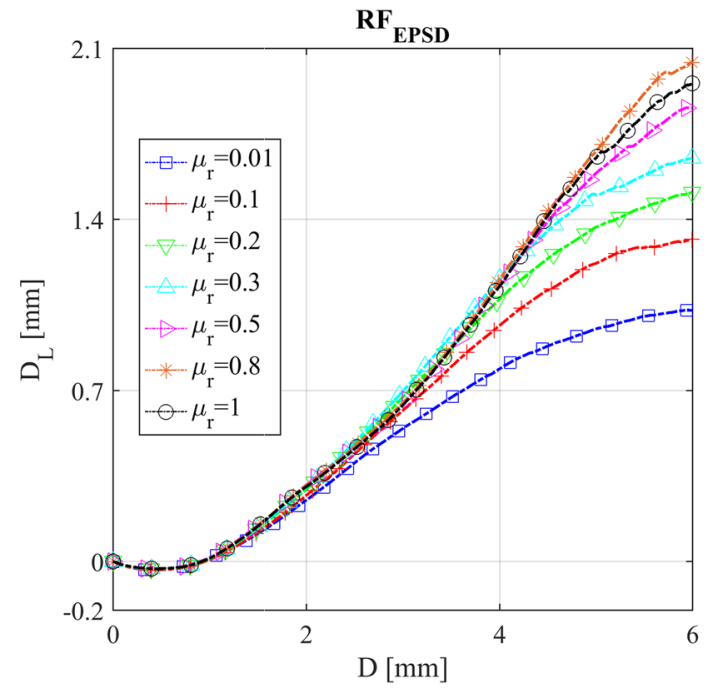

(c) 
Fig. 10 Vertical displacement of the lid during the shearing for loose packings a $\mathrm{RF}_{\mathrm{RVD}} \mathbf{b} \mathrm{RF}_{\mathrm{CI}} \mathbf{c} \mathrm{RF}_{\mathrm{EPSD}}$

for the first part of shearing and then starts to present a volume expansion (i.e. behaves approximately similar to a medium dense sample). Consequently, it can be said that although $\mathrm{RF}_{\mathrm{RVD}}$ contributes to the formation of looser packings, it is unable to provide particles with dilative behaviour during the shearing process once $\mu_{r}>0.01$.

Moreover, it can be seen that $\mathrm{RF}_{\mathrm{CI}}$ is approximately following $\mathrm{RF}_{\mathrm{RVD}}$ (except for higher volume expansion when $0.01 \leq \mu_{r} \leq 0.1$ ). Meanwhile, packings with $\mathrm{RF}_{\mathrm{EPSD}}$ provide a different response compared to those of $\mathrm{RF}_{\mathrm{RVD}}$ and $\mathrm{RF}_{\mathrm{CI}}$; regardless of the initial porosity, all the samples (except $\left.\mu_{r}=0.01\right)$ present a half-half contractive/dilative behaviour (i.e. until the half of the total shearing distance, samples are compressed and once a threshold for $\phi$ is reached, dilation is initiated), which represents characteristics of a mediumdense sample.

\subsection{Shear strength under direct shear}

The curves of shear stress as a function of shear displacement $(D)$, for spherical particles, are shown in Figs. 11 and 12 . It can be seen that for dense packings, $R F_{R V D}$ has the following characteristics: a) there is a slight increase in maximum shear strength once rolling resistance is considered, but it saturates at $\mu_{r}=0.1$ (overall, the maximum shear strength is increased by $15 \%$ compared to $\left.\mu_{r}=0\right)$; b) for the initial stiffness and the residual strength, the effect of rolling resistance is indeed insignificant.

Additionally, for $\mu_{r}<0.3$, packings with $\mathrm{RF}_{\mathrm{CI}}$ have a similar shearing response to that of $\mu_{r}=0$. However, for larger values of $\mu_{r}$ a lower shear strength is observed. As mentioned earlier, for $\mathrm{RF}_{\mathrm{CI}}$ with $\mu_{r}>0.3$ sliding starts to happen among most of contacts, and accordingly the packing experiences a strength loss.

Results for $\mathrm{RF}_{\mathrm{EPSD}}$ are markedly different to those for $\mathrm{RF}_{\mathrm{CI}}$ and $\mathrm{RF}_{\mathrm{RVD}}$; increase of $\mu_{r}$ results in significantly higher shear strength for the dense samples and its effect saturates in the range $\mu_{r}=0.5 \ldots 0.8$. Additionally, the largest peak shear strength is almost two times the maximum shear strength for $\mathrm{RF}_{\mathrm{CI}}$ and $\mathrm{RF}_{\mathrm{RVD}}$. For dense packings, therefore, only $\mathrm{RF}_{\mathrm{EPSD}}$ has a significant influence on the shear strength of the granular assembly, which increases with increasing rolling friction value.

Figure 7 shows that rolling resistance contributes to the formation of loose packings. However, it is not clear whether applying rolling resistance for loose packings can influence the shearing response of the granular assemblies. Accordingly, the shearing response of the relatively loose packings is summarized in Fig. 12.

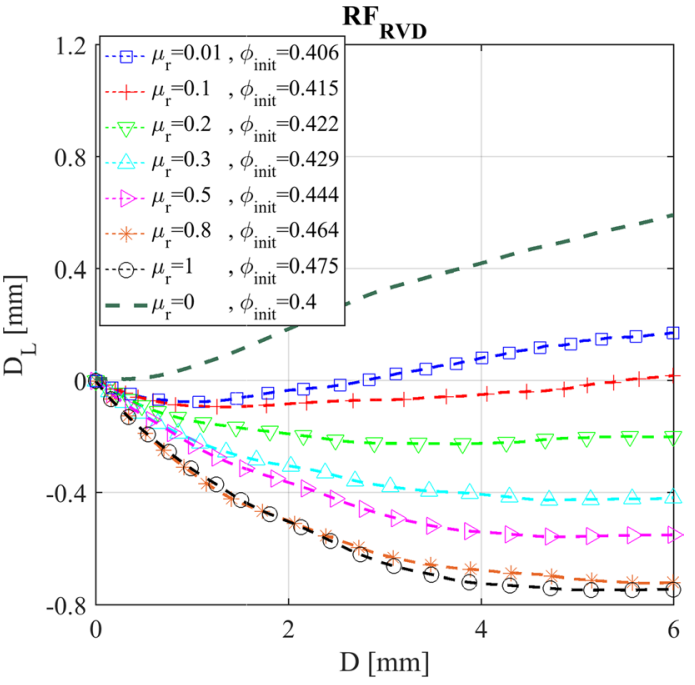

(a)

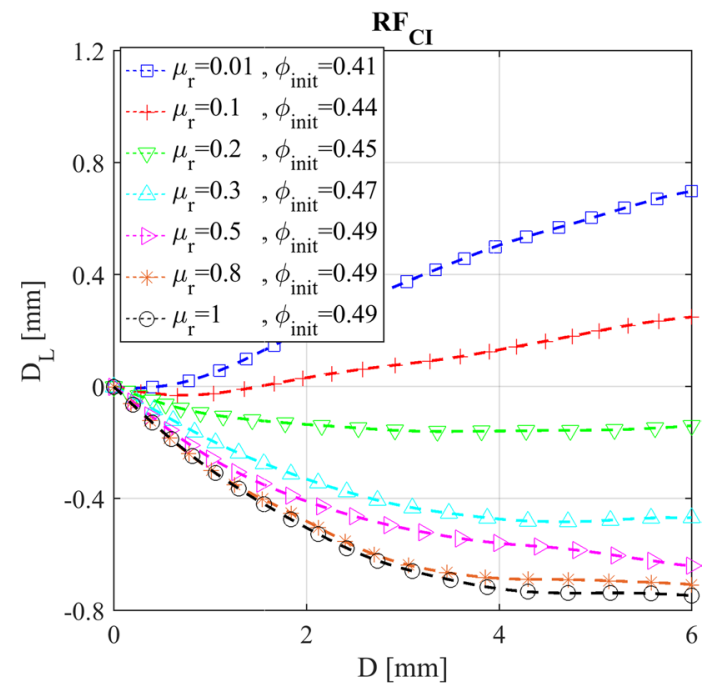

(b)

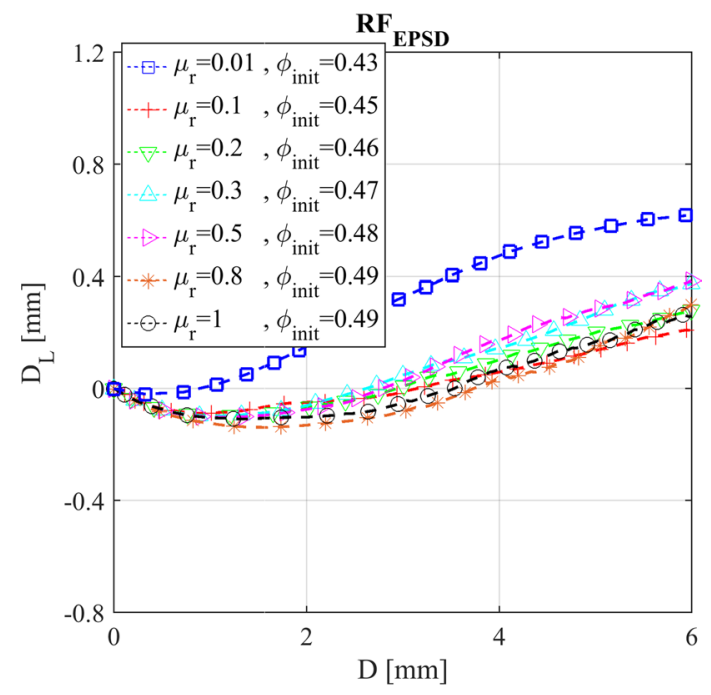

(c) 
Fig. 11 Shear stress-displacement curves considering various values of $\mu_{r}$, for dense packings $\mathbf{a} \mathrm{RF}_{\mathrm{RVD}} \mathbf{b} \mathrm{RF}_{\mathrm{CI}} \mathbf{c} \mathrm{RF}_{\mathrm{EPSD}}$

For both $\mathrm{RF}_{\mathrm{RVD}}$ and $\mathrm{RF}_{\mathrm{CI}}$, increasing $\mu_{r}$ decreases to a large extent both the initial stiffness and the maximum shear stress for the packings; nonetheless, the shear strength at critical state is approximately comparable. This reduction in shear strength can be partially related to lower packing density, however, it seems that these two models fail to calculate appropriate opposing torque once particles are compressed. This hypothesis arises from the dependence of the torque calculation on the normal force magnitude; here, due to the presence of higher normal forces in a shear cell, the calculated torque is over-predicted for these models, which forces the particle to mobilize by sliding.

Some kinks are observed for $\mathrm{RF}_{\mathrm{CI}}$ and $\mathrm{RF}_{\mathrm{RVD}}$ models, at intermediate shear displacement in Fig. 12. This can be justified by the fact that the volume contraction, happening during the shearing in loose samples, can slightly contribute to an increase in shear strength. A similar behaviour is seen in results provided by Wang and Gutierrez [31], and similarly reported by Jo et al. [18].

For packings with $\mathrm{RF}_{\mathrm{EPSD}}$ it is clear that, despite the initially loose packings, the shear strength is increased by increasing the rolling friction coefficient (up to $\mu_{r}=0.5$ ). This response is completely different from the observations for $\mathrm{RF}_{\mathrm{RVD}}$ and $\mathrm{RF}_{\mathrm{Cl}}$, which makes $\mathrm{RF}_{\mathrm{EPSD}}$ the only appropriate rolling resistance model for obtaining reasonable direct shear results (for both loose and dense packings).

A more detailed comparison is provided in Fig. 13 for the maximum friction angle of all the spherical packings. For dense packing, results from the three models are shown, however, the loose packings are only presented for $\mathrm{RF}_{\mathrm{EPSD}}$ (since both $\mathrm{RF}_{\mathrm{CI}}$ and $\mathrm{RF}_{\mathrm{RVD}}$ fail to influence the shearing response reasonably). It is seen that only $\mathrm{RF}_{\mathrm{EPSD}}$, with both loose and dense packing, increases its shear strength with $\mu_{r}$ variation.

Results provided in Sects. 4.1, 4.2 and 4.3 give an overall understanding regarding the applicability of the three rolling resistance models to simulate packing, shearing and dilative behaviour of non-spherical particles. It is observed that all three models can affect the density of packing. However, it must be noted that $\mathrm{RF}_{\mathrm{CI}}$ and $\mathrm{RF}_{\mathrm{RVD}}$ models are not suitable for simulation of the dense shearing regimes (i.e. direct shear test). This can be justified by the fact that calculated torque in $\mathrm{RF}_{\mathrm{CI}}$ and $\mathrm{RF}_{\mathrm{RVD}}$ is a direct function of normal contact force, and no limitation is applied to gauge a maximum torque value. Since in direct shear test, an external vertical force is acting through the lid, a higher normal contact force, and thus a high torque value is foreseen. A high value of $\mu_{r}$ cancels the rotational degree of freedom. Therefore, for both

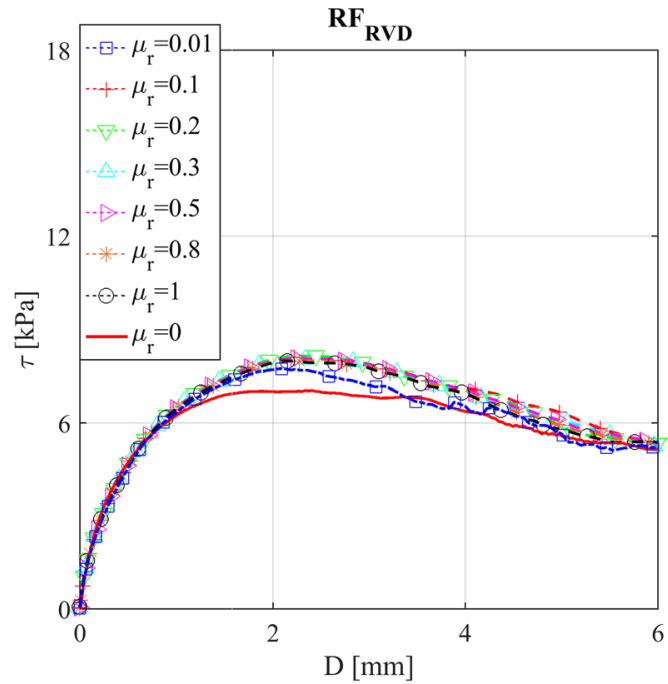

(a)

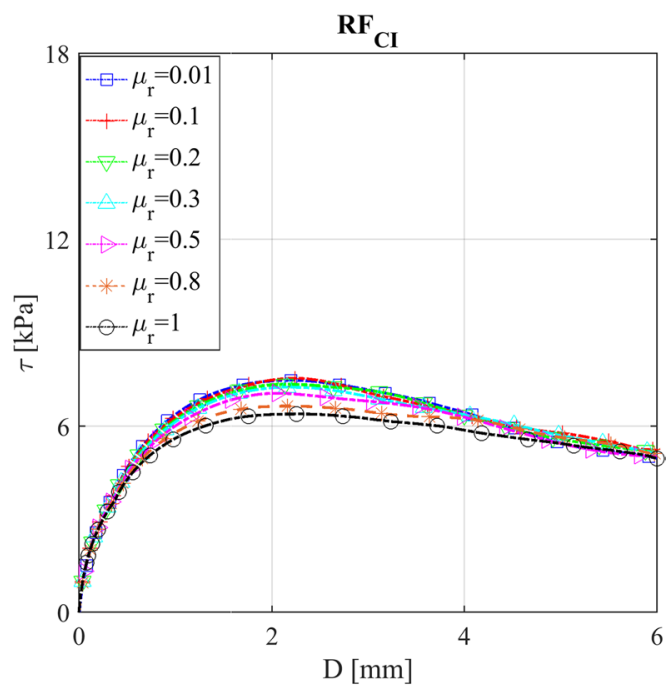

(b)

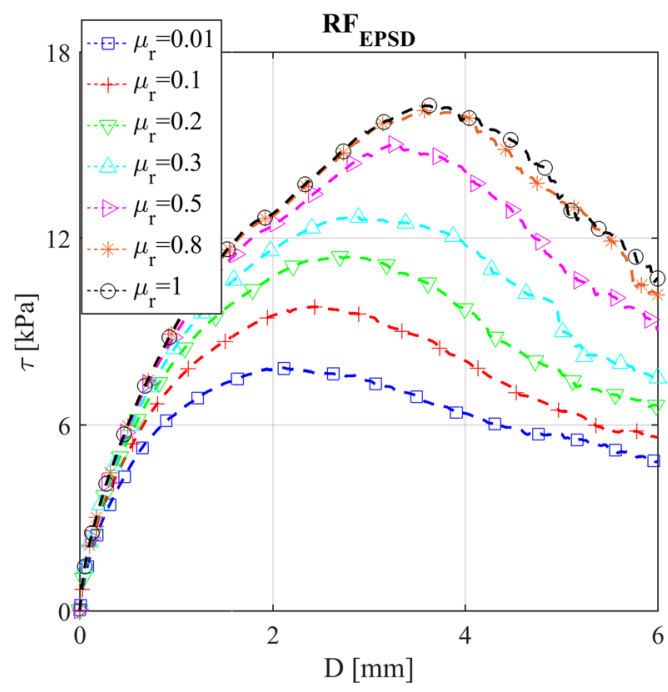

(c) 


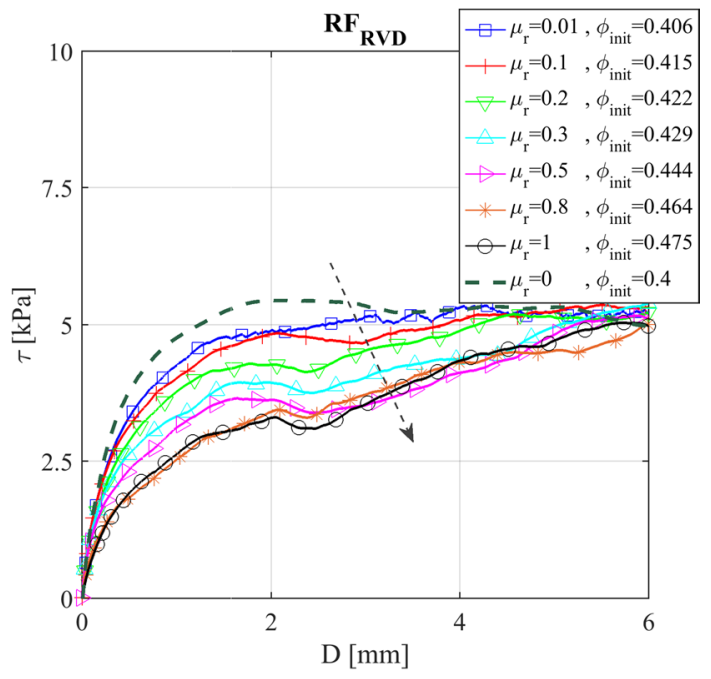

(a)

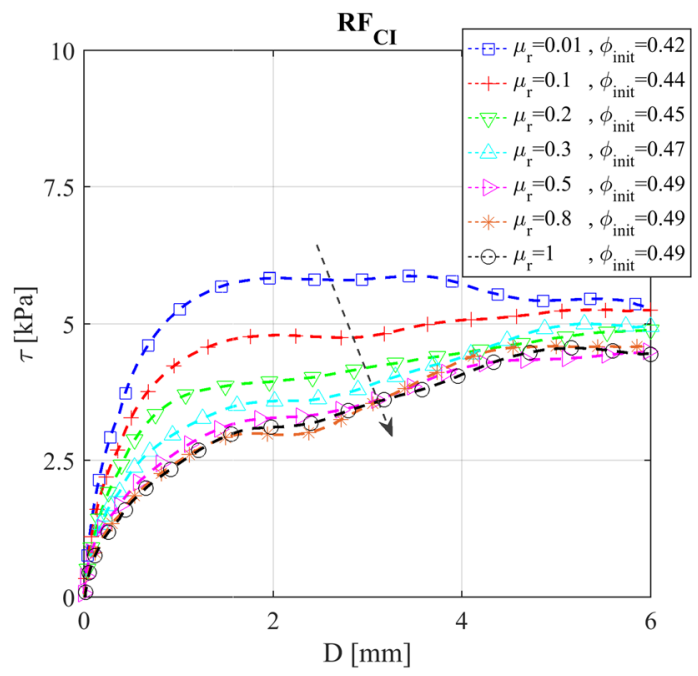

(b)

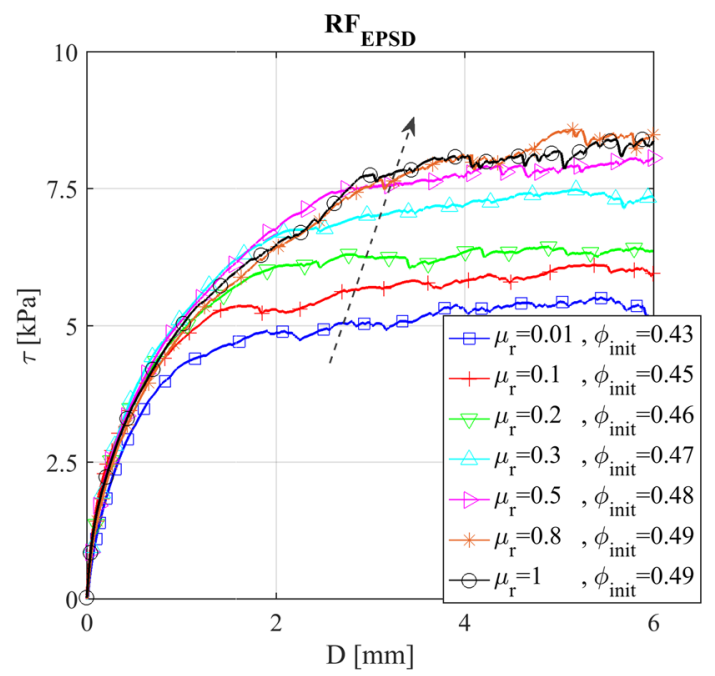

(c)

Fig. 12 Shear stress-displacement curves considering various values of $\mu_{r}$, for loose packings $\mathbf{a} \mathrm{RF}_{\mathrm{RVD}} \mathbf{b} \mathrm{RF}_{\mathrm{CI}} \mathbf{c} \mathrm{RF}_{\mathrm{EPSD}}$

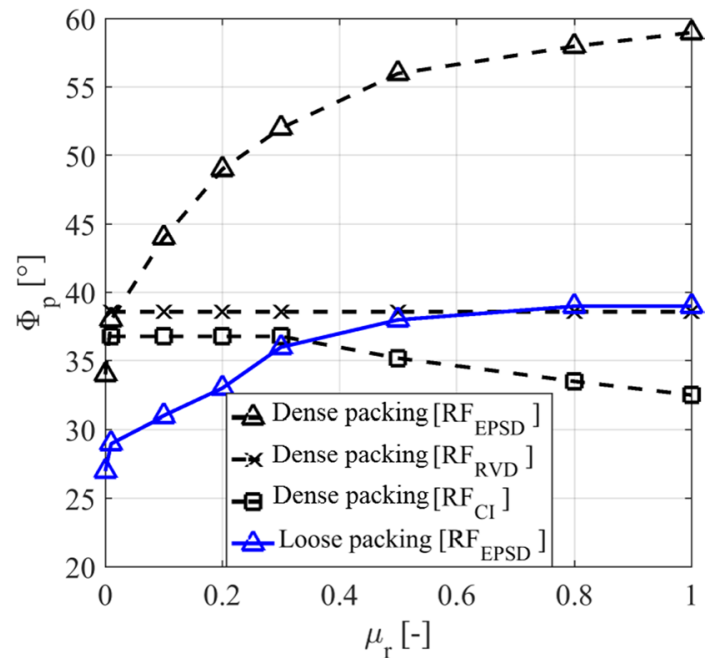

Fig. 13 Summary of the peak friction angle for various packing densities (for $\mathrm{RF}_{\mathrm{RVD}}, \mathrm{RF}_{\mathrm{CI}}$ and $\mathrm{RF}_{\mathrm{EPSD}}$ )

rolling resistance models sliding starts to happen among most contacts and accordingly the packing experiences a non-physical change in strength and dilation (this is not in agreement with mechanical expectations from shape factor). Conversely, $\mathrm{RF}_{\mathrm{EPSD}}$ can be applied for estimating shear strength and dilative response of the non-spherical granular assembly.

Non-spherical particles Similar to Sect. 3, the effect of aspect ratio on cuboids and cylindrical particles (with various surface and edge properties) is investigated within the shear cell, see Fig. 14 . The peak friction angle $\left(\Phi_{p}\right)$ of the packings, which is obtained through dividing the maximum value of normal and shear stresses, is plotted with respect to $A R$. For SQ cuboids, $\Phi_{p}$ increases sharply for $\mathrm{SQ}(N 4)$ up to $A R=1.5$, whereas for $\mathrm{SQ}(N 6)$ and $\mathrm{SQ}(N 8)$ the change in $\Phi_{p}$ is smaller and limited by $A R=1.25$, see Fig. 14a. However, MS cuboids show less dependency on the $A R$ value (only MS(27) shows an increase for $A R=1.25$ ).

On the other hand, cylindrical particle with $N=2$ and $A R=1$ (i.e. spheres) have the lowest $\Phi_{p}$, which is significantly increased at $A R=1.25$ (i.e. converting to ellipsoid), see Fig. 14b. For $A R>1.25$ results for $\mathrm{SQ}(N 2)$ and $\mathrm{SQ}(N 4)$ overlap. Other cylindrical particles with sharper edges only show a small change in $\Phi_{p}$ for $A R=1.25$ (no changes are seen for $A R>1.25$ ).

\section{Discussion}

In this section results from angle-of-repose and direct-shear tests are compared for spherical and non-spherical particles. Considering AoR results for cuboidal and cylindrical 


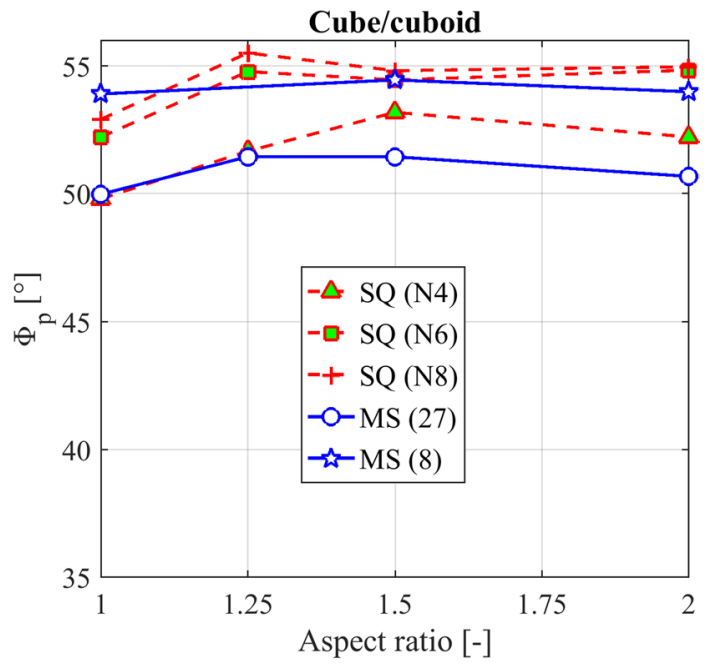

(a)

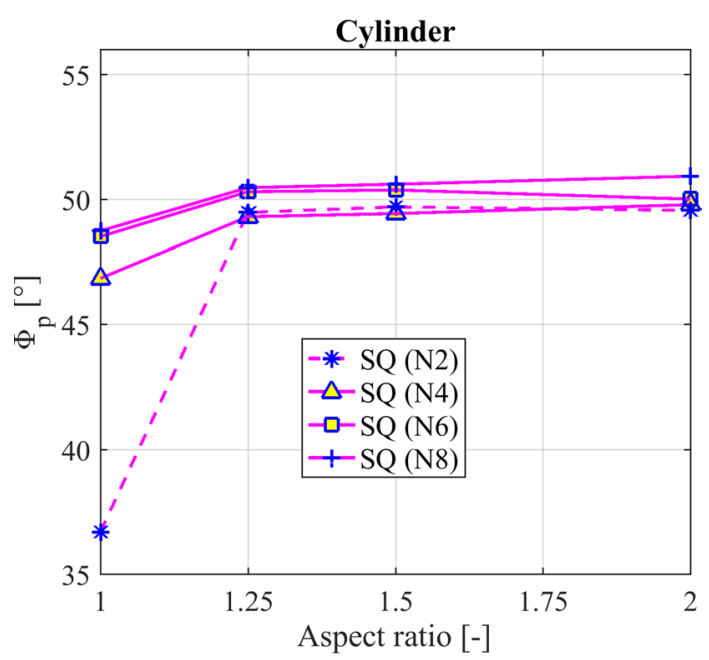

(b)

Fig. 14 Dependence of peak friction angle on aspect ratio for a cube/ cuboids $\mathbf{b}$ cylinders

particles with various $A R$ and comparing them with those of spherical particles (accompanied by three rolling resistance models), the following can be pointed out: a) $\mathrm{RF}_{\mathrm{RVD}}$ and $\mathrm{RF}_{\mathrm{EPSD}}$ can provide a similar AoR to that of $A R=1$ for cubes with $0.2<\mu_{r}<0.4$ and for cylinders with $0.1<\mu_{r}<0.2$ (see Fig. 15), b) for $A R>1$, to compensate a similar AoR (for both cuboid and cylinders), spherical particles with $\mathrm{RF}_{\mathrm{RVD}}$ and $\mathrm{RF}_{\mathrm{EPSD}}$ need to have a maximum value of $\mu_{r}=\mu_{s}=0.56$ (it is usually suggested that rolling resistance coefficient must be smaller/equal to sliding friction coefficient) c) for $\mathrm{RF}_{\mathrm{Cl}}$, it can be seen that assigning $0.08<\mu_{r}<0.18$ can provide AoR values comparable to all range of $A R$ (for both cubes and cylinders). Consequently, it is clarified in this section that for the angle-of-repose test, the incorporation of rolling resistance models can well mimic the avalanching response of non-spherical particles.

Furthermore, the shear strength for cubes/cuboids and cylinders is compared with spherical particles. As already discussed, only $\mathrm{RF}_{\mathrm{EPSD}}$ provides an approximately monotonic increase for the peak friction angle of spherical assemblies (i.e. with increase of $\mu_{r}$ ). Accordingly, the peak friction angle of the spherical packings with $\mathrm{RF}_{\mathrm{EPSD}}$ is summarized in Fig. 16. Results in Fig. 16 suggest that dense packings with $\mathrm{RF}_{\mathrm{EPSD}}$ can be calibrated (by varying $\mu_{r}$ ) to obtain $\Phi_{p}$ values similar to that obtained for non-spherical particles.

\section{Conclusions}

The current work compares the avalanching, packing, shearing and dilative response for spherical particles (in presence of three different rolling resistance models) with common non-spherical particles with aspect ratios of one and higher (cylinders and cubes/cuboids). The non-spherical shapes are simulated by multi-sphere (MS) and superquadric (SQ) shape representation methods, each of which has unique shape complexity properties (MS particles have an inherent surface pseudo-bumpiness, whereas SQ approach can well-produce sharp edges). Accordingly, the ability of the artificial shape representation techniques (i.e. rolling resistance models) to simulate a wide range of shape complexity parameters is evaluated.

For the AoR test, results suggest that tuning the rolling friction parameter $\mu_{r}$, as a shape parameter for spheres (considering all three models), can lead to the desired heap characteristics (which is comparable to the results of nonspherical particles with various bumpiness, blockiness and aspect ratios).

For the direct shear test, it is seen that including rolling resistance can provide a wide range of initial porosities $\phi_{\text {init }}$. However, it is seen that spherical packings provide always a higher $\phi_{\text {init }}$ than cubes and cylinders.

Additionally, the capability of the considered rolling resistance models in providing dilative response, as it happens for non-spherical particles during shearing, is evaluated. Results suggest that $\mathrm{RF}_{\mathrm{RVD}}$ and $\mathrm{RF}_{\mathrm{CI}}$ are addressing this phenomenon only for dense packing. However, $\mathrm{RF}_{\mathrm{EPSD}}$ is well capable of providing dilation for the granular assemblies almost for the entire range of packing densities (which is in agreement with results reported in [29], where it is shown that even loose samples of non-spherical particles tend to dilate).

For the shear strength, once $\mu_{r}$ is varied for packings with various $\phi_{\text {init }}$, only $\mathrm{RF}_{\mathrm{EPSD}}$ provides $\Phi_{p}$ that is well matching with those obtained for cubical/cuboidal and cylindrical 
Legend: $\mathbf{1}-1.7$ Cubes/cuboids $\quad$ Cylinder

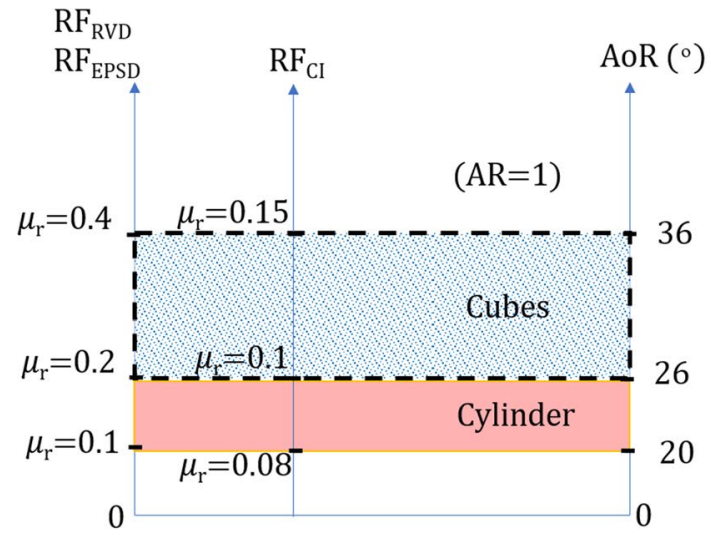

(a)

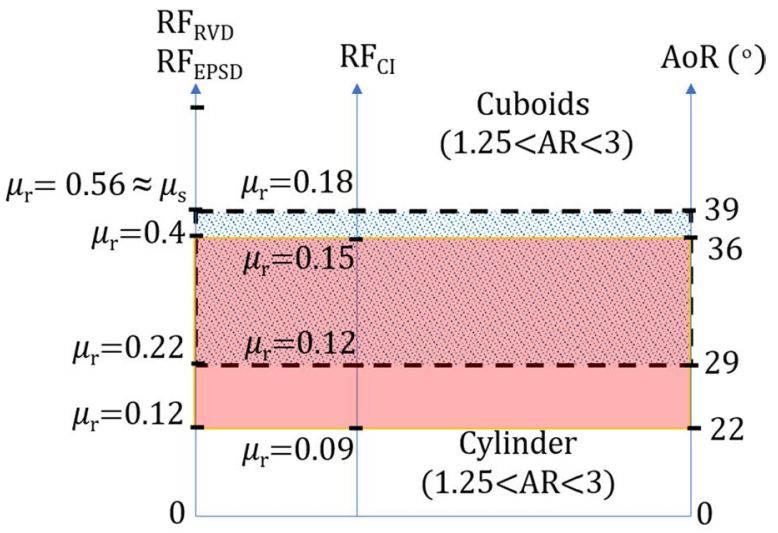

(b)

Fig. 15 Graphical description of equivalent $\mu_{r}$ for obtaining similar AoR values with a cubes and cylinders with AR=1 b cuboids and cylinders with $\mathrm{AR}>1$

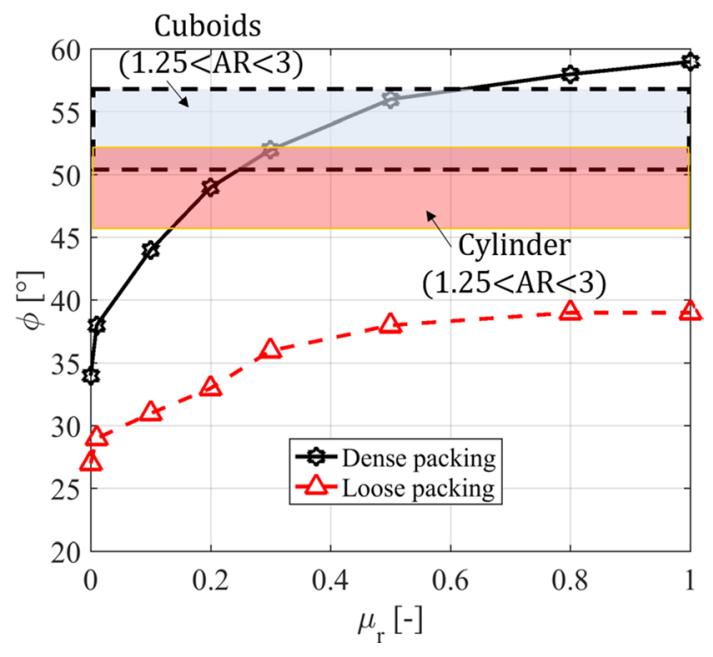

Fig. 16 Graphical description of equivalent $\mu_{r}$ for obtaining similar peak friction angle to cuboid and cylinder particles

shape particles. The issue associated with $R F_{C D T}$ and $\mathrm{RF}_{\mathrm{CI}}$, in case of dilative response and shear strength, has been explored further. It is proposed that since the calculated torque is dependent on normal force magnitude, which is high in case of shear test, the calculated torque is over-predicted. Restricting rotational freedom of the spheres results in pure sliding of particles (this deficiency is less prominent for dense packings, where due to dense arrangement of particles sliding is happening the least).

In summary, the results for $\mathrm{RF}_{\mathrm{EPSD}}$ suggested that it is possible to calibrate the rolling friction $\mu_{r}$ to obtain the desired individual macro-scale response of non-spherical particles (e.g. angle of repose and shear strength).
Meanwhile, results clearly showed the defects of both $\mathrm{RF}_{\mathrm{CI}}$ for $\mathrm{RF}_{\mathrm{RVD}}$ models in a dense shearing regime.

A future study should consider the influence of polydispersity on bulk response of non-spherical particles, as well as consider a loose initial packing with different rolling friction only during the shearing (similar to that applied to dense packings).

Acknowledgements This work has been carried out as a part of the T-MAPPP project, an EU-funded Framework 7 Marie Curie Initial Training Network under Grant agreement No. ITN 607453. The financial support provided by the European Union is gratefully acknowledged.

\section{Compliance with ethical standards}

Conflict of interest The authors declare that they have no conflict of interest.

Open Access This article is licensed under a Creative Commons Attribution 4.0 International License, which permits use, sharing, adaptation, distribution and reproduction in any medium or format, as long as you give appropriate credit to the original author(s) and the source, provide a link to the Creative Commons licence, and indicate if changes were made. The images or other third party material in this article are included in the article's Creative Commons licence, unless indicated otherwise in a credit line to the material. If material is not included in the article's Creative Commons licence and your intended use is not permitted by statutory regulation or exceeds the permitted use, you will need to obtain permission directly from the copyright holder. To view a copy of this licence, visit http://creativecommons.org/licenses/by/4.0/. 


\section{References}

1. Ai, J., Chen, J.F., Rotter, M., Ooi, J.Y.: Assessment of rolling resistance models in discrete element simulations. Powder Technol. 206(3), 269-282 (2011). https://doi.org/10.1016/j.powte c. 2010.09 .030

2. Aigner, A., Schneiderbauer, S., Kloss, C., Pirker, S.: Determining the coefficient of friction by shear tester simulation. In: 3rd Int. Conf. on Particle-Based Methods, vol. 1, pp. 335-342 (2013)

3. Anand, A., Curtis, J.S., Wassgren, C.R., Hancock, B.C., Ketterhagen, W.R.: Predicting discharge dynamics from a rectangular hopper using the discrete element method (DEM). Chem. Eng. Sci. 63(24), 5821-5830 (2008)

4. Aste, T.: Variations around disordered close packing. J. Phys. Condens. Matter 17(24), S2361 (2005)

5. Bardet, J., Huang, Q.: Rotational stiffness of cylindrical particle contacts. Powders Grains 93, 39-43 (1993)

6. Barr, A.H.: Superquadrics and angle-preserving transformations. IEEE Comput. Graph. Appl. 1(January), 11-23 (1981). https:// doi.org/10.1109/MCG.1981.1673799

7. Brilliantov, N.V., Spahn, F., Hertzsch, J.M., Pöschel, T.: Model for collisions in granular gases. Phys. Rev. E 53(5), 5382 (1996)

8. Chen, H., Zhao, S., Zhou, X.: DEM investigation of angle of repose for super-ellipsoidal particles. Particuology 50, 53-66 (2019)

9. DEM Solutions Ltd.: EDEM 2.6 theory reference guide. Tech. rep. (2014)

10. Estrada, N., Azéma, E., Radjai, F., Taboada, A.: Identification of rolling resistance as a shape parameter in sheared granular media. Phys. Rev. E 84(1), 11306 (2011)

11. Estrada, N., Taboada, A., Radjaï, F.: Shear strength and force transmission in granular media with rolling resistance. Phys. Rev. E 78(2), 21301 (2008). https://doi.org/10.1103/PhysR evE.78.021301

12. Favier, J., Abbaspourfard, M., Kremmer, M., Raji, A.: Shape representation of axisymmetrical, nonspherical particles in discrete element simulation using multielement model particles. Eng. Comput. 16(4), 467-480 (1999). https://doi.org/10.1108/02644 409910271894

13. Härtl, J., Ooi, J.Y.: Numerical investigation of particle shape and particle friction on limiting bulk friction in direct shear tests and comparison with experiments. Powder Technol. 212(1), 231-239 (2011)

14. Iwashita, K., Oda, M.: Rolling resistance at contacts in simulation of shear band development by DEM. J. Eng. Mech. 124(3), 285-292 (1998)

15. Jerier, J.F., Richefeu, V., Imbault, D., Donzé, F.V.: Packing spherical discrete elements for large scale simulations. Comput. Methods Appl. Mech. Eng. 199(25-28), 1668-1676 (2010)

16. Jiang, M., Shen, Z., Wang, J.: A novel three-dimensional contact model for granulates incorporating rolling and twisting resistances. Comput. Geotech. 65, 147-163 (2015)

17. Jiang, M.J., Yu, H.S., Harris, D.: A novel discrete model for granular material incorporating rolling resistance. Comput. Geotech. 32(5), 340-357 (2005)

18. Jo, S.A., Kim, E.K., Cho, G.C., Lee, S.W.: Particle shape and crushing effects on direct shear behavior using DEM. Soils Found. 51(4), 701-712 (2011)

19. Kasina, V.P.R.: DEM modelling and quantitative validation of flow characteristics and blending of pellets in a planar silo (2016)

20. Kesseler, M., Heller, V., Turnbull, B.: A laboratory-numerical approach for modelling scale effects in dry granular slides.
Landslides 15(11), 2145-2159 (2018). https://doi.org/10.1007/ s10346-018-1023-Z

21. Kloss, C., Goniva, C., Hager, A., Amberger, S., Pirker, S.: Models, algorithms and validation for opensource DEM and CFDDEM. Prog. Comput. Fluid Dyn. Int. J. 12(2/3), 140 (2012). https://doi.org/10.1504/PCFD.2012.047457

22. Ma, Z., Wang, Y., Ren, N., Shi, W.: A coupled CFD-DEM simulation of upward seepage flow in coarse sands. Marine Georesources Geotechnol. 37(5), 589-598 (2019)

23. Mindlin, R.D., Deresiewicz, H.: Elastic spheres in contact under varying oblique forces. J. Appl. Mech. 327, 327-344 (1953)

24. Miura, K., Maeda, K., Toki, S.: Method of measurement for the angle of repose of sands. Soils Found. 37(2), 89-96 (1997)

25. Nguyen, D.H., Azéma, É., Radjai, F., Sornay, P.: Effect of size polydispersity versus particle shape in dense granular media. Phys. Rev. E 90(1), 12202 (2014)

26. Podlozhnyuk, A., Pirker, S., Kloss, C.: Efficient implementation of superquadric particles in discrete element method within an open-source framework. Comput. Part. Mech. 4(1), 101-118 (2017). https://doi.org/10.1007/s40571-016-0131-6

27. Seelen, L.J.H., Padding, J.T., Kuipers, J.A.M.: A granular discrete element method for arbitrary convex particle shapes: method and packing generation. Chem. Eng. Sci. 189, 84-101 (2018)

28. Soltanbeigi, B., Podlozhnyuk, A., Ooi, J.Y., Kloss, C., Papanicolopulos, S.A.: Comparison of multi-sphere and superquadric particle representation for modelling shearing and flow characteristics of granular assemblies. In: EPJ Web of Conferences, vol. 140, p. 6015. EDP Sciences (2017)

29. Soltanbeigi, B., Podlozhnyuk, A., Papanicolopulos, S.A., Kloss, C., Pirker, S., Ooi, J.Y.: DEM study of mechanical characteristics of multi-spherical and superquadric particles at micro and macro scales. Powder Technol. 329, 288-303 (2018)

30. Suhr, B., Six, K.: On the effect of stress dependent interparticle friction in direct shear tests. Powder Technol. 294, 211-220 (2016)

31. Wang, J., Gutierrez, M.: Discrete element simulations of direct shear specimen scale effects. Géotechnique 60(5), 395-409 (2010)

32. Wensrich, C., Katterfeld, A.: Rolling friction as a technique for modelling particle shape in DEM. Powder Technol. 217, 409-417 (2012). https://doi.org/10.1016/j.powtec.2011.10.057

33. Zhao, S., Evans, T.M., Zhou, X.: Shear-induced anisotropy of granular materials with rolling resistance and particle shape effects. Int. J. Solids Struct. 150, 268-281 (2018)

34. Zhou, B., Huang, R., Wang, H., Wang, J.: DEM investigation of particle anti-rotation effects on the micromechanical response of granular materials. Granul. Matter 15(3), 315-326 (2013)

35. Zhou, C., Ooi, J.Y.: Numerical investigation of progressive development of granular pile with spherical and non-spherical particles. Mech. Mater. 41(6), 707-714 (2009). https://doi. org/10.1016/j.mechmat.2009.01.017

36. Zhou, Y., Wang, H., Zhou, B., Li, J.: DEM-aided direct shear testing of granular sands incorporating realistic particle shape. Granul. Matter 20(3), 55 (2018)

37. Zhou, Y.C., Wright, B.D., Yang, R.Y., Xu, B.H., Yu, A.B.: Rolling friction in the dynamic simulation of sandpile formation. Physica A Stat. Mech. Its Appl. 269(2), 536-553 (1999)

Publisher's Note Springer Nature remains neutral with regard to jurisdictional claims in published maps and institutional affiliations. 